\title{
All-order renormalization of propagator matrix for unstable Dirac fermions
}

\author{
Bernd A. Kniehl \\ Kavli Institute for Theoretical Physics, University of California, \\ Santa Barbara, California 93106-4030, USA \\ (Received 6 March 2014; published 13 May 2014)
}

\begin{abstract}
We consider a system of unstable Dirac fermions in a general parity-nonconserving theory with intergeneration mixing and explain how to renormalize its propagator matrix to all orders in perturbation theory. We work in the pole scheme, in which the squares of the renormalized masses are identified with the complex pole positions and the wave-function renormalization (WFR) matrices are adjusted according to the Lehmann-Symanzik-Zimmermann reduction formalism. The unit-residue property is explicitly verified for the renormalized dressed propagator matrix. Closed analytic expressions for the pole-mass counterterms and WFR matrices in terms of the self-energy functions are presented. We identify residual degrees of freedom in the WFR matrices and propose an additional renormalization condition to exploit them. We demonstrate that, in the presence of instability, the WFR matrices of the in and out states bifurcate in the sense that they are no longer related by pseudo-Hermitian conjugation. The well-known one- and two-loop results for stable fermions are recovered. The all-order renormalized propagator of a single unstable fermion takes a particularly compact form. We also briefly discuss Dirac spinors for unstable fermions.

\section{INTRODUCTION}

The standard model (SM) of elementary particle physics has been enormously consolidated by the recent discovery at the CERN Large Hadron Collider of a weak neutral resonance that shares all its properties with the SM Higgs boson within the experimental precision [1], in the complete absence of signals of physics beyond the SM. In view of the present uncertainty in the pole mass of the top quark [2], it is even possible for the SM vacuum to be stable way up to the scale of the Planck mass [3].

On the theoretical side, this provides a strong motivation for us to deepen and complete our understanding of the SM as a renormalizable quantum field theory [4]. The on-shell renormalization scheme, which includes the physical particle masses and Sommerfeld's fine-structure constant among the basic parameters, provides a natural framework for that. It was systematically elaborated at one loop for stable particles in Refs. [5-7], and a particularly useful variant of it was proposed prior to that in Ref. [8]. Using the algebraic method, it was generalized to all orders of perturbation theory assuming all particles to be stable, taking the neutrinos to be massless, and neglecting quark-flavor mixing [9].

The incorporation of mixing and instability of elementary particles in the renormalization of the SM requires generalized concepts for flavor-changing propagators and vertices. In the SM with massless neutrinos, these are the propagator matrices of the up- and down-type quarks and

\footnotetext{
*Permanent address: II. Institut für Theoretische Physik, Universität Hamburg, Luruper Chaussee 149, 22761 Hamburg, Germany.
}

their charged-current vertices, which involve the CabibboKobayashi-Maskawa (CKM) [10] quark mixing matrix. This pattern carries over to the lepton sector if the neutrinos are massive Dirac fermions, and the analogue of the CKM matrix is the Pontecorvo-Maki-Nakagawa-Sakata [11] neutrino mixing matrix. Things are more complicated in the presence of Majorana degrees of freedom in the neutrino sector, which typically give rise to flavor-changing vertices involving the $Z^{0}$ and Higgs bosons, too.

Various renormalization prescriptions for mixing matrices of Dirac [12-14] and Majorana [15,16] fermions were proposed in the literature, some of which naturally extend to all orders. As pointed out in Ref. [13], necessary conditions for the renormalized fermion mixing matrices include UV finiteness, gauge independence, and (pseudo) unitarity. Furthermore, it is desirable for their counterterms to be on shell, flavor democratic, finite in case of fermion mass degeneracy, and expressible in terms of self-energies only [14]. As for the renormalization of the CKM matrix, comparative numerical analyses were performed for the partial widths of the hadronic $W$-boson decays [17] and the top-quark decays [18].

As for the renormalization of propagator matrices of mixed systems of fermions, the situation is as follows. In Ref. [19], the pole masses were shown to be gauge independent to all orders in the SM using Nielsen identities [20], both for stable and unstable fermions. In Ref. [21], the renormalization of the propagator matrix of stable Dirac fermions was studied, including also wave-function renormalization (WFR), and it was explicitly proven that the WFR conditions proposed by Aoki, Hioki, Kawabe, Konuma, and Muta (AHKKM) [6] guarantee the unitresidue properties of the diagonal elements of the 
renormalized propagator matrix to all orders, in compliance with the Lehmann-Symanzik-Zimmermann (LSZ) reduction formalism [22]. In a very recent letter [23], the discussion of Ref. [21] was extended to the case of unstable Dirac fermions, and closed expressions for their mass counterterms and WFR matrices valid to all orders of perturbation theory were listed. The purpose of this paper is to explain the derivation of these expressions in detail and to expose their anatomy.

This paper is organized as follows. In Sec. II, we start from the inverse of the unrenormalized propagator matrix and obtain the dressed propagator matrix by performing the Dyson resummation [24]. At this point, we define the renormalization conditions for the complex pole masses in terms of secular equations. In Sec. III, we introduce the WFR matrices, explain how they enter the dressed propagator matrix, and define renormalized self-energies in such a way that the renormalized propagator matrix emerges from its unrenormalized counterpart by replacing the unrenormalized self-energies in the latter by their renormalized counterparts. In Sec. IV, we generalize the AHKKM WFR conditions [6] to the case of instability and impose them on the inverse of the renormalized propagator matrix obtained in Sec. III. In Sec. V, we exactly solve the system of equations derived in Sec. IV for the WFR matrices, so as to establish them in closed analytic form valid to all orders of perturbation theory. In doing so, we observe that the generalized AHKKM renormalization conditions do not completely fix the WFR matrices and propose an additional renormalization condition to exhaust this residual freedom. A similar observation was made for the case of stability at one loop [15]. The generalized AHKKM renormalization conditions also allow us to exactly solve the secular equations mentioned above, so as to obtain closed analytic expressions for the pole-mass counterterms to all orders of perturbation theory. In Sec. VI, we recover a phenomenon that was previously encountered in Ref. [25] at one loop and that was named WFR bifurcation in Ref. [23]. In Sec. VII, we explicitly prove that the generalized AHKKM renormalization conditions ensure that, if the mass shell of a Dirac fermion is reached, the respective diagonal element of the renormalized propagator matrix resonates with unit residue, in accordance with the LSZ reduction formalism [22]. In Sec. VIII, we study the special case of a solitary Dirac fermion and find a particularly compact form for its renormalized propagator. In Sec. IX, we apply the formalism developed in Secs. II-V to the one-loop case and show how the well-known one-loop results for the cases of stability [15] and instability [25] are recovered. Section $X$ contains a summary and an outlook. In Appendix A, we collect a few theorems of matrix algebra that are used in our derivations. In Appendix B, we introduce Dirac spinors for unstable fermions and discuss their properties.

\section{UNRENORMALIZED DRESSED PROPAGATOR MATRIX}

We consider a system of $N$ unstable Dirac fermions in the context of some general parity-nonconserving theory with intergeneration mixing, such as the up-type or down-type quarks in the SM. We denote the bare quantum fields of their flavor eigenstates by $\psi_{i}^{\prime 0}(x)$, where the subscript $i=1, \ldots, N$ is the flavor index and the superscript 0 labels bare quantities. For the sake of a compact notation, we group them into a column vector in flavor space,

$$
\Psi^{\prime 0}(x)=\left(\begin{array}{c}
\psi_{1}^{\prime 0}(x) \\
\vdots \\
\psi_{N}^{\prime 0}(x)
\end{array}\right) .
$$

While flavor mixing is already built into the bare Lagrangian via appropriate interaction terms, such as the charged-current vertices in the SM, instability is implemented by introducing the concept of complex pole mass via the renormalization procedure leaving the bare masses real. Therefore, the construction of the unrenormalized dressed propagator of a mixed system of unstable Dirac fermions is the same as in the case of stability, which was discussed in Sec. II of Ref. [21]. For the reader's convenience and to set the stage for the subsequent elaboration of the renormalization procedure, we summarize the relevant results of Ref. [21] in the following, albeit in a somewhat different notation, which is more appropriate for our purposes here.

The kinetic term of the bare Lagrangian is

$$
\mathcal{L}^{0}(x)=\bar{\Psi}^{\prime 0}(x)\left(i \varnothing-\mathcal{M}^{\prime 0}\right) \Psi^{\prime 0}(x),
$$

where $\bar{\Psi}^{\prime 0}(x)=\left[\Psi^{\prime 0}(x)\right]^{\dagger} \gamma^{0}$ and $\mathcal{M}^{\prime 0}$ is the bare mass matrix. In order for $\mathcal{L}^{0}(x)$ to be Hermitian, $\mathcal{M}^{\prime 0}$ must satisfy the pseudo-Hermiticity relation $\gamma^{0} \mathcal{M}^{\prime 0 \dagger} \gamma^{0}=\mathcal{M}^{\prime 0}$, which constrains it to the form $\mathcal{M}^{\prime 0}=M^{\prime 0} a_{+}+M^{\prime 0 \dagger} a_{-}$, where $M^{\prime 0}$ is an arbitrary complex $N \times N$ matrix and $a_{ \pm}=\left(I_{4} \pm \gamma_{5}\right) / 2$ are the chiral projection operators. Here and in the following, $I_{n}$ denotes the $n \times n$ unit matrix. By the singular-value decomposition theorem, $M^{\prime 0}$ may be transformed into a real diagonal matrix $M^{0}$ with nonnegative entries, ${ }^{1}$

$$
M_{i j}^{0}=m_{i}^{0} \delta_{i j}
$$

by a biunitary transformation $M^{0}=U_{-} M^{\prime 0} U_{+}^{\dagger}$, where $U_{ \pm} U_{ \pm}^{\dagger}=U_{ \pm}^{\dagger} U_{ \pm}=I_{N}$. The bare field multiplet of the mass eigenstates $\psi_{i}^{0}(x)$, with bare masses $m_{i}^{0}$, is then given by $\Psi^{0}(x)=U \Psi^{\prime 0}(x)$, where $U=U_{+} a_{+}+U_{-} a_{-}$. Upon

\footnotetext{
${ }^{1}$ In this paper, summation over repeated indices is not implied in the absence of summation symbols.
} 
this field transformation, Eq. (2) indeed assumes the standard form

$$
\begin{aligned}
\mathcal{L}^{0}(x)= & \bar{\Psi}^{0}(x)\left(i \partial-M^{0}\right) \Psi^{0}(x) \\
= & i \bar{\Psi}_{+}^{0}(x) \partial \Psi_{+}^{0}(x)+i \bar{\Psi}_{-}^{0}(x) \partial \Psi_{-}^{0}(x) \\
& -\bar{\Psi}_{+}^{0}(x) M^{0} \Psi_{-}^{0}(x)-\bar{\Psi}_{-}^{0}(x) M^{0} \Psi_{+}^{0}(x),
\end{aligned}
$$

where $\Psi_{ \pm}^{0}(x)=a_{ \pm} \Psi^{0}(x)$ and $\bar{\Psi}_{ \pm}^{0}(x)=\bar{\Psi}^{0}(x) a_{\mp}$.

In momentum space, the unrenormalized propagator matrix is defined as $i P(\not p)=\int d^{4} x e^{i p \cdot x}$ $\times\left\langle 0\left|T\left[\Psi^{0}(x) \bar{\Psi}^{0}(0)\right]\right| 0\right\rangle$, where $T$ is the time-ordered product and a tensorial product both in the spinor and generation spaces is implied. Its inverse is built up by the one-particleirreducible Feynman diagrams contributing to the transitions $j \rightarrow i$. We have

$$
P^{-1}(\not p)=\not p-M^{0}-\Sigma(\not p),
$$

where $\Sigma(\not p)$ is the unrenormalized self-energy matrix. By Lorentz covariance, the latter has the form

$$
\Sigma(\not p)=\left[\not p B_{+}\left(p^{2}\right)+A_{+}\left(p^{2}\right)\right] a_{+}+(+\leftrightarrow-),
$$

where the entries in the matrices $A_{ \pm}\left(p^{2}\right)$ and $B_{ \pm}\left(p^{2}\right)$ are Lorentz-invariant functions of $p^{2}$. The latter may be calculated from the bare Lagrangian order by order in perturbation theory. However, we refrain from resorting to perturbative expansions in the following rendering our results valid to all orders. Defining

$$
S_{ \pm}\left(p^{2}\right)=I_{N}-B_{ \pm}\left(p^{2}\right), \quad T_{ \pm}\left(p^{2}\right)=M^{0}+A_{ \pm}\left(p^{2}\right),
$$

Eq. (5) may be cast into a compact form,

$$
P^{-1}(\not p)=\left[p S_{+}\left(p^{2}\right)-T_{+}\left(p^{2}\right)\right] a_{+}+(+\leftrightarrow-) .
$$

Performing the Dyson resummation [24] is equivalent to inverting Eq. (8) and yields [21]

$$
\begin{aligned}
P(\not p)= & {\left[\not p+D_{-}\left(p^{2}\right)\right] S_{-}^{-1}\left(p^{2}\right)\left[p^{2}-E_{-}\left(p^{2}\right)\right]^{-1} a_{+} } \\
& +(+\leftrightarrow-) \\
= & a_{+}\left[p^{2}-F_{+}\left(p^{2}\right)\right]^{-1} S_{+}^{-1}\left(p^{2}\right)\left[\not p+C_{+}\left(p^{2}\right)\right] \\
& +(+\leftrightarrow-),
\end{aligned}
$$

with the shorthand notations

$C_{ \pm}\left(p^{2}\right)=T_{\mp}\left(p^{2}\right) S_{\mp}^{-1}\left(p^{2}\right), \quad D_{ \pm}\left(p^{2}\right)=S_{\mp}^{-1}\left(p^{2}\right) T_{ \pm}\left(p^{2}\right)$,

$E_{ \pm}\left(p^{2}\right)=C_{ \pm}\left(p^{2}\right) C_{\mp}\left(p^{2}\right), \quad F_{ \pm}\left(p^{2}\right)=D_{\mp}\left(p^{2}\right) D_{ \pm}\left(p^{2}\right)$

In fact, Eqs. (8) and (9) are easily seen to satisfy $P(\not p) P^{-1}(\not p)=P^{-1}(\not p) P(\not p)=I_{N} \otimes I_{4}$, where $\otimes$ denotes the tensorial product. From the first equality in Eq. (7) it follows that $\operatorname{det}\left[S_{ \pm}\left(p^{2}\right)\right]=1+\mathcal{O}(\alpha) \neq 0$ with $\alpha$ being a generic coupling constant, so that we may use Eq. (A7) to evaluate $S_{ \pm}^{-1}\left(p^{2}\right)$ as $S_{ \pm}^{-1}\left(p^{2}\right)=\operatorname{adj}\left[S_{ \pm}\left(p^{2}\right)\right] / \operatorname{det}\left[S_{ \pm}\left(p^{2}\right)\right]$. Alternatively, we may compute $S_{ \pm}^{-1}\left(p^{2}\right)$ perturbatively as a geometric series, $S_{ \pm}^{-1}\left(p^{2}\right)=I_{N}+\sum_{n=1}^{\infty} B_{ \pm}^{n}\left(p^{2}\right)$.

Since the four matrices $\left[p^{2}-E_{ \pm}\left(p^{2}\right)\right]$ and $\left[p^{2}-F_{ \pm}\left(p^{2}\right)\right]$, whose inverses appear in the individual propagator parts in Eq. (9), are related by similarity transformations,

$$
\begin{aligned}
p^{2}-E_{+}\left(p^{2}\right) & =C_{-}\left(p^{2}\right)\left[p^{2}-E_{-}\left(p^{2}\right)\right] C_{-}^{-1}\left(p^{2}\right) \\
& =S_{+}\left(p^{2}\right)\left[p^{2}-F_{+}\left(p^{2}\right)\right] S_{+}^{-1}\left(p^{2}\right) \\
& =T_{-}\left(p^{2}\right)\left[p^{2}-F_{-}\left(p^{2}\right)\right] T_{-}^{-1}\left(p^{2}\right),
\end{aligned}
$$

we have

$$
\begin{aligned}
\operatorname{det}\left[p^{2}-E_{+}\left(p^{2}\right)\right] & =\operatorname{det}\left[p^{2}-E_{-}\left(p^{2}\right)\right] \\
& =\operatorname{det}\left[p^{2}-F_{+}\left(p^{2}\right)\right] \\
& =\operatorname{det}\left[p^{2}-F_{-}\left(p^{2}\right)\right],
\end{aligned}
$$

which, by virtue of Eq. (A7), guarantees that the individual propagator parts all have poles at the same (complex) positions $p^{2}=M_{i}^{2}$ defined as the zeros of Eq. (12) by any of the secular equations $[19,21,26]$

$$
\operatorname{det}\left[M_{i}^{2}-E_{ \pm}\left(M_{i}^{2}\right)\right]=\operatorname{det}\left[M_{i}^{2}-F_{ \pm}\left(M_{i}^{2}\right)\right]=0 .
$$

Here, $M_{i}$ is the complex pole mass of the Dirac fermion $i$. It is related to the real pole mass $m_{i}$ and total decay width $\Gamma_{i}$ as $[27,28]$

$$
M_{i}=m_{i}-i \frac{\Gamma_{i}}{2}
$$

In the Appendix of Ref. [21], Eq. (13) is solved perturbatively through two loops for the case of stability. In the remainder of this section, we derive closed all-order expressions for $M_{i}$ in terms of the Lorentz-invariant functions $\left[A_{ \pm}\left(p^{2}\right)\right]_{i j}$ and $\left[B_{ \pm}\left(p^{2}\right)\right]_{i j}$. Owing to Eq. (A1), we have the identities

$$
\begin{aligned}
& \left\{\left[p^{2}-F_{ \pm}\left(p^{2}\right)\right] \operatorname{adj}\left[p^{2}-F_{ \pm}\left(p^{2}\right)\right]\right\}_{i i}=\operatorname{det}\left[p^{2}-F_{ \pm}\left(p^{2}\right)\right] \\
& \left\{\operatorname{adj}\left[p^{2}-E_{ \pm}\left(p^{2}\right)\right]\left[p^{2}-E_{ \pm}\left(p^{2}\right)\right]\right\}_{i i}=\operatorname{det}\left[p^{2}-E_{ \pm}\left(p^{2}\right)\right] .
\end{aligned}
$$

At this point, we introduce the two matrices,

$$
\begin{aligned}
& M_{i j}^{ \pm}=\left\{\operatorname{adj}\left[M_{j}^{2}-F_{ \pm}\left(M_{j}^{2}\right)\right]\right\}_{i j}, \\
& \bar{M}_{i j}^{ \pm}=\left\{\operatorname{adj}\left[M_{i}^{2}-E_{ \pm}\left(M_{i}^{2}\right)\right]\right\}_{i j},
\end{aligned}
$$

which we shall need again in Sec. $\mathrm{V}$, and observe that $M_{i i}^{ \pm}, \bar{M}_{i i}^{ \pm}=\prod_{j \neq i}\left(M_{i}^{2}-M_{j}^{2}\right)+\mathcal{O}(\alpha) \neq 0$. For the solution $p^{2}=M_{i}^{2}$ of Eq. (13), we then obtain from Eq. (15) that 


$$
M_{i}^{2}=\frac{\left[F_{ \pm}\left(M_{i}^{2}\right) M^{ \pm}\right]_{i i}}{M_{i i}^{ \pm}}=\frac{\left[\bar{M}^{ \pm} E_{ \pm}\left(M_{i}^{2}\right)\right]_{i i}}{\bar{M}_{i i}^{ \pm}}
$$

In Sec. V, we shall see that the WFR procedure generates yet another closed all-order expression for $M_{i}$, namely the one specified in Eq. (65), in which both matrices of Eq. (16) enter in a symmetric way.

\section{RENORMALIZED DRESSED PROPAGATOR MATRIX}

In the following, we adopt the pole renormalization scheme, in which the complex pole masses $M_{i}$ serve as the renormalized masses, i.e. the mass counterterms $\delta M_{i}$ are fixed by the relations

$$
m_{i}^{0}=M_{i}+\delta M_{i}
$$

The field renormalization is implemented by writing

$$
\Psi^{0}(x)=Z^{1 / 2} \Psi(x), \quad \bar{\Psi}^{0}(x)=\bar{\Psi}(x) \bar{Z}^{1 / 2},
$$

where $\Psi(x)$ is the renormalized field multiplet and

$$
Z^{1 / 2}=Z_{+}^{1 / 2} a_{+}+Z_{-}^{1 / 2} a_{-}, \quad \bar{Z}^{1 / 2}=a_{-} \bar{Z}_{+}^{1 / 2}+a_{+} \bar{Z}_{-}^{1 / 2},
$$

with $Z_{ \pm}^{1 / 2}$ and $\bar{Z}_{ \pm}^{1 / 2}$ being the WFR matrices. As already pointed out in Ref. [25] in the context of a one-loop analysis, we have to allow $\bar{Z}^{1 / 2}$ to differ from $\gamma^{0} Z^{\dagger 1 / 2} \gamma^{0}$,

$$
\bar{Z}^{1 / 2} \neq \gamma^{0} Z^{\dagger 1 / 2} \gamma^{0}
$$

This will be explicitly demonstrated in Sec. VI by means of closed all-order expressions for $\left(Z_{ \pm}^{1 / 2}\right)_{i j}$ and $\left(\bar{Z}_{ \pm}^{1 / 2}\right)_{i j}$ in terms of the functions $\left[A_{ \pm}\left(p^{2}\right)\right]_{i j}$ and $\left[B_{ \pm}\left(p^{2}\right)\right]_{i j}$ derived in Sec. V by requiring that the diagonal elements of the renormalized propagator matrix $[\hat{P}(p))]_{i i}$ have unit residues on their mass shells $p^{2}=M_{i}^{2}$. That the unit-residue condition cannot be satisfied using $Z^{\dagger 1 / 2}$ may be observed already in the unmixed case [28]. In fact, in the case of a single unstable Dirac fermion, the use of $Z_{ \pm}^{* 1 / 2}$ does not allow one to arrange for the parts of the renormalized propagator proportional to $a_{+}$and $a_{-}$to have both unit residues; one ends up with a relative phase factor between them, which becomes unity only in the zero-width limit [28]. A detailed discussion of this will be presented in Sec. VIII.

Solving Eq. (19) for the renormalized field multiplets, we have

$$
\Psi(x)=Z^{-1 / 2} \Psi^{0}(x), \quad \bar{\Psi}(x)=\bar{\Psi}^{0}(x) \bar{Z}^{-1 / 2},
$$

where

$$
\begin{aligned}
& Z^{-1 / 2}=Z_{+}^{-1 / 2} a_{+}+Z_{-}^{-1 / 2} a_{-}, \\
& \bar{Z}^{-1 / 2}=a_{-} \bar{Z}_{+}^{-1 / 2}+a_{+} \bar{Z}_{-}^{-1 / 2}
\end{aligned}
$$

are the inverses of the matrices in Eq. (20). Using Eq. (22), we may express the renormalized propagator matrix $i \hat{P}(\not p)=\int d^{4} x e^{i p \cdot x}\langle 0|T[\Psi(x) \bar{\Psi}(0)]| 0\rangle$ in terms of the unrenormalized one as

$$
\hat{P}(\not p)=Z^{-1 / 2} P(\not p) \bar{Z}^{-1 / 2}
$$

Substituting Eq. (9) into Eq. (24), we thus obtain

$$
\begin{aligned}
\hat{P}(\not p)= & {\left[Z_{-}^{-1 / 2} \not p+Z_{+}^{-1 / 2} D_{-}\left(p^{2}\right)\right] S_{-}^{-1}\left(p^{2}\right) } \\
& \times\left[p^{2}-E_{-}\left(p^{2}\right)\right]^{-1} \bar{Z}_{-}^{-1 / 2} a_{+}+(+\leftrightarrow-) \\
= & a_{+} Z_{+}^{-1 / 2}\left[p^{2}-F_{+}\left(p^{2}\right)\right]^{-1} S_{+}^{-1}\left(p^{2}\right) \\
& \times\left[p \bar{Z}_{+}^{-1 / 2}+C_{+}\left(p^{2}\right) \bar{Z}_{-}^{-1 / 2}\right]+(+\leftrightarrow-) .
\end{aligned}
$$

We may absorb the WFR matrices in Eq. (25) by defining renormalized counterparts of $S_{ \pm}\left(p^{2}\right)$ and $T_{ \pm}\left(p^{2}\right)$ in Eq. (7) as

$\hat{S}_{ \pm}\left(p^{2}\right)=\bar{Z}_{ \pm}^{1 / 2} S_{ \pm}\left(p^{2}\right) Z_{ \pm}^{1 / 2}, \quad \hat{T}_{ \pm}\left(p^{2}\right)=\bar{Z}_{\mp}^{1 / 2} T_{ \pm}\left(p^{2}\right) Z_{ \pm}^{1 / 2}$.

In analogy to Eq. (10), we are thus led to define

$\hat{C}_{ \pm}\left(p^{2}\right)=\hat{T}_{\mp}\left(p^{2}\right) \hat{S}_{\mp}^{-1}\left(p^{2}\right)=\bar{Z}_{ \pm}^{1 / 2} C_{ \pm}\left(p^{2}\right) \bar{Z}_{\mp}^{-1 / 2}$,

$\hat{D}_{ \pm}\left(p^{2}\right)=\hat{S}_{\mp}^{-1}\left(p^{2}\right) \hat{T}_{ \pm}\left(p^{2}\right)=Z_{\mp}^{-1 / 2} D_{ \pm}\left(p^{2}\right) Z_{ \pm}^{1 / 2}$,

$\hat{E}_{ \pm}\left(p^{2}\right)=\hat{C}_{ \pm}\left(p^{2}\right) \hat{C}_{\mp}\left(p^{2}\right)=\bar{Z}_{ \pm}^{1 / 2} E_{ \pm}\left(p^{2}\right) \bar{Z}_{ \pm}^{-1 / 2}$,

$\hat{F}_{ \pm}\left(p^{2}\right)=\hat{D}_{\mp}\left(p^{2}\right) \hat{D}_{ \pm}\left(p^{2}\right)=Z_{ \pm}^{-1 / 2} F_{ \pm}\left(p^{2}\right) Z_{ \pm}^{1 / 2}$.

Thus, Eq. (25) becomes

$$
\begin{aligned}
\hat{P}(\not p)= & {\left[\not p+\hat{D}_{-}\left(p^{2}\right)\right] \hat{S}_{-}^{-1}\left(p^{2}\right)\left[p^{2}-\hat{E}_{-}\left(p^{2}\right)\right]^{-1} a_{+} } \\
& +(+\leftrightarrow-) \\
= & a_{+}\left[p^{2}-\hat{F}_{+}\left(p^{2}\right)\right]^{-1} \hat{S}_{+}^{-1}\left(p^{2}\right)\left[\not p+\hat{C}_{+}\left(p^{2}\right)\right] \\
& +(+\leftrightarrow-) .
\end{aligned}
$$

By observing that

$$
\begin{aligned}
& \operatorname{det}\left[p^{2}-\hat{E}_{ \pm}\left(p^{2}\right)\right]=\operatorname{det}\left[p^{2}-E_{ \pm}\left(p^{2}\right)\right], \\
& \operatorname{det}\left[p^{2}-\hat{F}_{ \pm}\left(p^{2}\right)\right]=\operatorname{det}\left[p^{2}-F_{ \pm}\left(p^{2}\right)\right],
\end{aligned}
$$

we understand that the pole positions $M_{i}^{2}$ are not affected by the WFR, as it should be [19]. Mutatis mutandis, the inverse of the renormalized propagator matrix reads 


$$
\begin{aligned}
\hat{P}^{-1}(p) & =\left[p \hat{S}_{+}\left(p^{2}\right)-\hat{T}_{+}\left(p^{2}\right)\right] a_{+}+(+\leftrightarrow-) \\
& =\left[\bar{Z}_{+}^{1 / 2} p S_{+}\left(p^{2}\right)-\bar{Z}_{-}^{1 / 2} T_{+}\left(p^{2}\right)\right] Z_{+}^{1 / 2} a_{+}+(+\leftrightarrow-) .
\end{aligned}
$$

\section{GENERALIZED WFR CONDITIONS}

We now establish on-shell WFR conditions appropriate for the case of instability by requiring that the diagonal elements $[\hat{P}(\not p)]_{i i}$ of the renormalized propagator matrix have unit residues on their mass shells $p^{2}=M_{i}^{2}$, in accordance with the LSZ reduction formalism [22]. In the case of a mixed system of stable Dirac fermions, this may be achieved by imposing the on-shell WFR conditions specified in Eqs. (3.53a)-(3.54b) of Ref. [6]. Detailed inspection reveals that their derivation, as outlined in Ref. [6], carries over to the case of instability. An explicit proof of this will be presented in Sec. VII.

For the reader's convenience, we repeat here the derivation of the AHKKM WFR conditions [6] for the case of instability in our notation. Let us consider the limit $p^{2} \rightarrow M_{n}^{2}$ in which the Dirac fermion $n$ approaches its mass shell. The LSZ [22] renormalization condition on $\hat{P}(\not p)$,

$$
[\hat{P}(\not p)]_{i j}=\frac{\delta_{i n} \delta_{n j}}{\not p-M_{n}}+\mathcal{O}(1),
$$

necessitates that $\hat{P}^{-1}(\not p)$ behaves as

$$
\begin{aligned}
& {\left[\hat{P}^{-1}(\not p)\right]_{i j}} \\
& \quad= \begin{cases}\left(\not p-M_{n}\right)\left[I_{4}+\mathcal{O}\left(\not p-M_{n}\right)\right] & \text { if } i=n=j, \\
{\left[M_{i n}+\mathcal{O}\left(\not p-M_{n}\right)\right]\left(\not p-M_{n}\right)} & \text { if } i \neq n=j, \\
\left(\not p-M_{n}\right)\left[M_{n j}+\mathcal{O}\left(\not p-M_{n}\right)\right] & \text { if } i=n \neq j, \\
M_{i j}+\mathcal{O}\left(\not p-M_{n}\right) & \text { if } i \neq n \neq j,\end{cases}
\end{aligned}
$$

where $M_{i j}$ are constant matrices in four-dimensional spinor space, which, in general, do not commute with $p$. In fact, they are linear combinations of the Dirac matrices $I_{4}$ and $\gamma_{5}$ with constant coefficients. The specific structure of Eq. (32) may be easily understood by multiplying Eqs. (31) and (32) in both orders. The behavior in Eq. (32) may be arranged for by imposing the generalized version of the on-shell WFR conditions,

$$
\begin{gathered}
{\left[\hat{P}^{-1}(\not p)\right]_{i j} u\left(\vec{p}, M_{j}\right)=0,} \\
\bar{u}\left(\vec{p}, M_{i}\right)\left[\hat{P}^{-1}(\not p)\right]_{i j}=0, \\
\left\{\frac{1}{\not p-M_{i}}\left[\hat{P}^{-1}(\not p)\right]_{i i}\right\} u\left(\vec{p}, M_{i}\right)=u\left(\vec{p}, M_{i}\right),
\end{gathered}
$$

$$
\bar{u}\left(\vec{p}, M_{i}\right)\left\{\left[\hat{P}^{-1}(\not p)\right]_{i i} \frac{1}{p-M_{i}}\right\}=\bar{u}\left(\vec{p}, M_{i}\right),
$$

for $i, j=1, \ldots, N$. Here, $u\left(\vec{p}, M_{i}\right)$ and $\bar{u}\left(\vec{p}, M_{i}\right)$ are fourcomponent spinors satisfying the Dirac equations

$$
\begin{aligned}
& \left(\not p-M_{i}\right) u\left(\vec{p}, M_{i}\right)=0, \\
& \bar{u}\left(\vec{p}, M_{i}\right)\left(\not p-M_{i}\right)=0 .
\end{aligned}
$$

For the ease of notation, we have suppressed the spin labels in the arguments of the Dirac spinors. An elementary treatment of Dirac spinors for unstable fermions may be found in Appendix B.

Inserting Eq. (30) into Eqs. (33), (34), and (35), we obtain

$$
\begin{gathered}
{\left[\hat{S}_{\mp}\left(M_{j}^{2}\right)\right]_{i j} M_{j}-\left[\hat{T}_{ \pm}\left(M_{j}^{2}\right)\right]_{i j}=0,} \\
M_{i}\left[\hat{S}_{ \pm}\left(M_{i}^{2}\right)\right]_{i j}-\left[\hat{T}_{ \pm}\left(M_{i}^{2}\right)\right]_{i j}=0,
\end{gathered}
$$

and

$$
\begin{gathered}
\frac{1}{2}\left[\hat{S}_{+}\left(M_{i}^{2}\right)\right]_{i i}+M_{i}^{2}\left[\hat{S}_{+}^{\prime}\left(M_{i}^{2}\right)\right]_{i i}-M_{i}\left[\hat{T}_{+}^{\prime}\left(M_{i}^{2}\right)\right]_{i i} \\
+(+\leftrightarrow-)=1, \\
\frac{1}{2}\left[\hat{S}_{+}\left(M_{i}^{2}\right)\right]_{i i}-(+\leftrightarrow-)=0,
\end{gathered}
$$

respectively, while Eq. (36) is redundant. In Eq. (40), $f^{\prime}\left(M_{i}^{2}\right)=\left[d f\left(p^{2}\right) / d p^{2}\right]_{p^{2}=M_{i}^{2}}$. For $i=j$, Eqs. (38) and (39) imply that

$$
\begin{aligned}
M_{i}\left[\hat{S}_{+}\left(M_{i}^{2}\right)\right]_{i i} & =M_{i}\left[\hat{S}_{-}\left(M_{i}^{2}\right)\right]_{i i}=\left[\hat{T}_{+}\left(M_{i}^{2}\right)\right]_{i i} \\
& =\left[\hat{T}_{-}\left(M_{i}^{2}\right)\right]_{i i},
\end{aligned}
$$

which already contains Eq. (41).

\section{SOLUTION OF GENERALIZED WFR CONDITIONS}

We now solve Eqs. (38)-(40) for the WFR matrices $Z_{ \pm}^{1 / 2}$ and $\bar{Z}_{ \pm}^{1 / 2}$. Multiplying Eq. (38) by $\left[\hat{S}_{\mp}^{-1}\left(M_{j}^{2}\right)\right]_{k i}$ from the left and Eq. (39) by $\left[\hat{S}_{ \pm}^{-1}\left(M_{i}^{2}\right)\right]_{j k}$ from the right and summing over $i$ and $j$, respectively, we obtain

$$
\begin{aligned}
{\left[\hat{D}_{ \pm}\left(M_{j}^{2}\right)\right]_{i j} } & =\delta_{i j} M_{j}, \\
{\left[\hat{C}_{\mp}\left(M_{i}^{2}\right)\right]_{i j} } & =M_{i} \delta_{i j} .
\end{aligned}
$$

Iterating Eqs. (43) and (44), we get

$$
\left[\hat{F}_{ \pm}\left(M_{j}^{2}\right)\right]_{i j}=\delta_{i j} M_{j}^{2},
$$




$$
\left[\hat{E}_{ \pm}\left(M_{i}^{2}\right)\right]_{i j}=M_{i}^{2} \delta_{i j} .
$$

Using the last two equalities in Eq. (27), multiplying Eq. (45) by $\left(Z_{ \pm}^{1 / 2}\right)_{k i}$ from the left and Eq. (46) by $\left(\bar{Z}_{ \pm}^{1 / 2}\right)_{j k}$ from the right, and summing over $i$ and $j$, respectively, we find the following eigenvalue equations:

$$
\begin{aligned}
& {\left[F_{ \pm}\left(M_{j}^{2}\right) Z_{ \pm}^{1 / 2}\right]_{i j}=\left(Z_{ \pm}^{1 / 2}\right)_{i j} M_{j}^{2},} \\
& {\left[\bar{Z}_{ \pm}^{1 / 2} E_{ \pm}\left(M_{i}^{2}\right)\right]_{i j}=M_{i}^{2}\left(\bar{Z}_{ \pm}^{1 / 2}\right)_{i j} .}
\end{aligned}
$$

We may cast Eqs. (47) and (48) into the standard forms of homogeneous systems of linear equations, namely

$$
\begin{aligned}
& \left\{\left[M_{j}^{2}-F_{ \pm}\left(M_{j}^{2}\right)\right] Z_{ \pm}^{1 / 2}\right\}_{i j}=0, \\
& \left\{\bar{Z}_{ \pm}^{1 / 2}\left[M_{i}^{2}-E_{ \pm}\left(M_{i}^{2}\right)\right]\right\}_{i j}=0 .
\end{aligned}
$$

We need to solve Eq. (49) with fixed index $j$ for $\left(Z_{ \pm}^{1 / 2}\right)_{i j}$ and Eq. (50) with fixed index $i$ for $\left(\bar{Z}_{ \pm}^{1 / 2}\right)_{i j}$. Exploiting Eq. (13), we may glean from Eq. (A1) that the general solutions read

$$
\begin{aligned}
& \left(Z_{ \pm}^{1 / 2}\right)_{i j}=\sum_{k=1}^{N}\left\{\operatorname{adj}\left[M_{j}^{2}-F_{ \pm}\left(M_{j}^{2}\right)\right]\right\}_{i k}\left(\Lambda^{ \pm}\right)_{k j} \\
& \left(\bar{Z}_{ \pm}^{1 / 2}\right)_{i j}=\sum_{k=1}^{N}\left(\bar{\Lambda}^{ \pm}\right)_{i k}\left\{\operatorname{adj}\left[M_{i}^{2}-E_{ \pm}\left(M_{i}^{2}\right)\right]\right\}_{k j}
\end{aligned}
$$

where $\Lambda^{ \pm}$and $\bar{\Lambda}^{ \pm}$are constant matrices. It turns out to be sufficient to take the latter to be diagonal,

$$
\left(\Lambda^{ \pm}\right)_{i j}=\delta_{i j} \lambda_{j}^{ \pm}, \quad\left(\bar{\Lambda}^{ \pm}\right)_{i j}=\bar{\lambda}_{i}^{ \pm} \delta_{i j},
$$

where $\lambda_{j}^{ \pm}$and $\bar{\lambda}_{i}^{ \pm}$are constants yet to be determined. Inserting Eq. (52) into Eq. (51), we have

$$
\begin{aligned}
& \left(Z_{ \pm}^{1 / 2}\right)_{i j}=M_{i j}^{ \pm} \lambda_{j}^{ \pm}, \\
& \left(\bar{Z}_{ \pm}^{1 / 2}\right)_{i j}=\bar{\lambda}_{i}^{ \pm} \bar{M}_{i j}^{ \pm},
\end{aligned}
$$

where $M_{i j}^{ \pm}$and $\bar{M}_{i j}^{ \pm}$are defined in Eq. (16). Putting $i=j$ in Eqs. (53) and (54), we have

$$
\begin{aligned}
& \lambda_{j}^{ \pm}=\frac{\left(Z_{ \pm}^{1 / 2}\right)_{j j}}{M_{j j}^{ \pm}}, \\
& \bar{\lambda}_{i}^{ \pm}=\frac{\left(\bar{Z}_{ \pm}^{1 / 2}\right)_{i i}}{\bar{M}_{i i}^{ \pm}} .
\end{aligned}
$$

Feeding Eqs. (55) and (56) back into Eqs. (53) and (54), we may express the nondiagonal elements of the WFR matrices in terms of the diagonal ones as

$$
\begin{aligned}
\left(Z_{ \pm}^{1 / 2}\right)_{i j} & =\frac{M_{i j}^{ \pm}}{M_{j j}^{ \pm}}\left(Z_{ \pm}^{1 / 2}\right)_{j j}, \\
\left(\bar{Z}_{ \pm}^{1 / 2}\right)_{i j} & =\left(\bar{Z}_{ \pm}^{1 / 2}\right)_{i i} \frac{\bar{M}_{i j}^{ \pm}}{\bar{M}_{i i}^{ \pm}} .
\end{aligned}
$$

We may determine $\lambda_{j}^{ \pm}$and $\bar{\lambda}_{i}^{ \pm}$from Eqs. (40) and (42). To this end, we substitute Eqs. (53) and (54) into Eq. (26) and write

$$
\left[\hat{S}_{ \pm}\left(p^{2}\right)\right]_{i i}=\bar{\lambda}_{i}^{ \pm} s_{i}^{ \pm}\left(p^{2}\right) \lambda_{i}^{ \pm}, \quad\left[\hat{T}_{ \pm}\left(p^{2}\right)\right]_{i i}=\bar{\lambda}_{i}^{\mp} t_{i}^{ \pm}\left(p^{2}\right) \lambda_{i}^{ \pm},
$$

with

$s_{i}^{ \pm}\left(p^{2}\right)=\left[\bar{M}^{ \pm} S_{ \pm}\left(p^{2}\right) M^{ \pm}\right]_{i i}, \quad t_{i}^{ \pm}\left(p^{2}\right)=\left[\bar{M}^{\mp} T_{ \pm}\left(p^{2}\right) M^{ \pm}\right]_{i i}$.

Differentiating Eq. (59) with respect to $p^{2}$, we obtain

$$
\left[\hat{S}_{ \pm}^{\prime}\left(p^{2}\right)\right]_{i i}=\bar{\lambda}_{i}^{ \pm} s_{i}^{ \pm \prime}\left(p^{2}\right) \lambda_{i}^{ \pm}, \quad\left[\hat{T}_{ \pm}^{\prime}\left(p^{2}\right)\right]_{i i}=\bar{\lambda}_{i}^{\mp} t_{i}^{ \pm \prime}\left(p^{2}\right) \lambda_{i}^{ \pm},
$$

with

$$
\begin{aligned}
& s_{i}^{ \pm \prime}\left(p^{2}\right)=-\left[\bar{M}^{ \pm} B_{ \pm}^{\prime}\left(p^{2}\right) M^{ \pm}\right]_{i i}, \\
& t_{i}^{ \pm \prime}\left(p^{2}\right)=\left[\bar{M}^{\mp} A_{ \pm}^{\prime}\left(p^{2}\right) M^{ \pm}\right]_{i i},
\end{aligned}
$$

where we have used Eq. (7). Substituting Eqs. (59) and (61) into Eqs. (40) and (42), we obtain

$$
\begin{gathered}
M_{i} \bar{\lambda}_{i}^{+} s_{i}^{+} \lambda_{i}^{+}=M_{i} \bar{\lambda}_{i}^{-} s_{i}^{-} \lambda_{i}^{-}=\bar{\lambda}_{i}^{-} t_{i}^{+} \lambda_{i}^{+}=\bar{\lambda}_{i}^{+} t_{i}^{-} \lambda_{i}^{-}, \\
\bar{\lambda}_{i}^{+} s_{i}^{+} \lambda_{i}^{+}+M_{i}^{2}\left(\bar{\lambda}_{i}^{+} s_{i}^{+\prime} \lambda_{i}^{+}+\bar{\lambda}_{i}^{-} s_{i}^{-\prime} \lambda_{i}^{-}\right) \\
-M_{i}\left(\bar{\lambda}_{i}^{-} t_{i}^{+\prime} \lambda_{i}^{+}+\bar{\lambda}_{i}^{+} t_{i}^{-\prime} \lambda_{i}^{-}\right)=1
\end{gathered}
$$

Here and in the following, it is understood that the functions $s_{i}^{ \pm}, t_{i}^{ \pm}, s_{i}^{ \pm \prime}$, and $t_{i}^{ \pm \prime}$ are to be evaluated at $p^{2}=M_{i}^{2}$ if their arguments are omitted. From Eq. (63), we obtain

$$
M_{i}^{2}=f_{i}\left(M_{i}^{2}\right)
$$

where

$$
f_{i}\left(p^{2}\right)=\frac{t_{i}^{+}\left(p^{2}\right) t_{i}^{-}\left(p^{2}\right)}{s_{i}^{+}\left(p^{2}\right) s_{i}^{-}\left(p^{2}\right)},
$$

which is the exact all-order solution of Eq. (13). From Eqs. (18) and (65), we obtain the all-order mass counterterm as

$$
\delta M_{i}=m_{i}^{0}-\sqrt{f_{i}\left(M_{i}^{2}\right)} .
$$


Alternatively, we could have used Eq. (17) instead of Eq. (65). Using also Eq. (14) and taking real and imaginary parts, we have

$$
\begin{gathered}
m_{i}=\operatorname{Re} \sqrt{f_{i}\left(M_{i}^{2}\right)}=m_{i}^{0}-\operatorname{Re} \delta M_{i}, \\
-\frac{\Gamma_{i}}{2}=\operatorname{Im} \sqrt{f_{i}\left(M_{i}^{2}\right)}=-\operatorname{Im} \delta M_{i},
\end{gathered}
$$

where we have taken into account that the bare masses $m_{i}^{0}$ are real. By the same token, the imaginary part of $\delta M_{i}$ is UV finite, as is evident from Eq. (69). Pulling out the factor $\bar{\lambda}_{i}^{+} s_{i}^{+} \lambda_{i}^{+}$on the left-hand side of Eq. (64) and exploiting the three equalities of Eq. (63), we get

$$
\begin{aligned}
1 & =\bar{\lambda}_{i}^{+} s_{i}^{+} \lambda_{i}^{+}\left[1+M_{i}^{2}\left(\frac{s_{i}^{+\prime}}{s_{i}^{+}}+\frac{s_{i}^{-\prime}}{s_{i}^{-}}-\frac{t_{i}^{+\prime}}{t_{i}^{+}}-\frac{t_{i}^{-\prime}}{t_{i}^{-}}\right)\right] \\
& =\bar{\lambda}_{i}^{+} s_{i}^{+} \lambda_{i}^{+}\left[1-M_{i}^{2} \frac{f_{i}^{\prime}\left(M_{i}^{2}\right)}{f_{i}\left(M_{i}^{2}\right)}\right] \\
& =\bar{\lambda}_{i}^{+} s_{i}^{+} \lambda_{i}^{+}\left[1-f_{i}^{\prime}\left(M_{i}^{2}\right)\right],
\end{aligned}
$$

where we have used Eqs. (66) and (65) in the second and third equalities, respectively. Using Eqs. (55), (56), (63), and (70), we finally obtain

$$
\begin{aligned}
& \left(\bar{Z}_{ \pm}^{1 / 2}\right)_{i i}\left(Z_{ \pm}^{1 / 2}\right)_{i i}=\frac{\bar{M}_{i i}^{ \pm} M_{i i}^{ \pm}}{s_{i}^{ \pm}\left[1-f_{i}^{\prime}\left(M_{i}^{2}\right)\right]} \\
& \left(\bar{Z}_{\mp}^{1 / 2}\right)_{i i}\left(Z_{ \pm}^{1 / 2}\right)_{i i}=\frac{M_{i} \bar{M}_{i i}^{\mp} M_{i i}^{ \pm}}{t_{i}^{ \pm}\left[1-f_{i}^{\prime}\left(M_{i}^{2}\right)\right]}
\end{aligned}
$$

For each value of $i$, Eqs. (71) and (72) provide four complex equations for the four complex unknowns $\left(Z_{ \pm}^{1 / 2}\right)_{i i}$ and $\left(\bar{Z}_{ \pm}^{1 / 2}\right)_{i i}$. However, one of these equations is redundant. This may be understood by observing that, due to Eq. (65), the product of the two expressions in Eq. (71) coincides with that of those in Eq. (72). This allows us to express any three of the unknowns in terms of the fourth one. Picking out e.g. $\left(Z_{+}^{1 / 2}\right)_{i i}$, we have

$$
\begin{aligned}
\left(Z_{-}^{1 / 2}\right)_{i i} & =\frac{M_{i} s_{i}^{+} M_{i i}^{-}}{t_{i}^{-} M_{i i}^{+}}\left(Z_{+}^{1 / 2}\right)_{i i}, \\
\left(\bar{Z}_{+}^{1 / 2}\right)_{i i} & =\frac{\bar{M}_{i i}^{+} M_{i i}^{+}}{s_{i}^{+}\left[1-f_{i}^{\prime}\left(M_{i}^{2}\right)\right]} \frac{1}{\left(Z_{+}^{1 / 2}\right)_{i i}}, \\
\left(\bar{Z}_{-}^{1 / 2}\right)_{i i} & =\frac{M_{i} \bar{M}_{i i}^{-} M_{i i}^{+}}{t_{i}^{+}\left[1-f_{i}^{\prime}\left(M_{i}^{2}\right)\right]} \frac{1}{\left(Z_{+}^{1 / 2}\right)_{i i}},
\end{aligned}
$$

while $\left(Z_{+}^{1 / 2}\right)_{i i}$ remains undetermined. We may exploit this residual freedom by choosing e.g.

$$
\left(\bar{Z}_{+}^{1 / 2}\right)_{i i}=\left(Z_{+}^{1 / 2}\right)_{i i}=\left(\frac{\bar{M}_{i i}^{+} M_{i i}^{+}}{s_{i}^{+}\left[1-f_{i}^{\prime}\left(M_{i}^{2}\right)\right]}\right)^{1 / 2} \text {. }
$$

However, we are then stuck with $\left(\bar{Z}_{-}^{1 / 2}\right)_{i i} \neq\left(Z_{-}^{1 / 2}\right)_{i i}$.

Despite this residual freedom, the diagonal elements $[\hat{P}(\not p)]_{i i}$ of the renormalized propagator matrix in Eq. (28) are uniquely determined. This may be understood by observing that the left- and right-handed propagator parts in Eq. (25) have the generic form $Z_{\sigma}^{-1 / 2} X \bar{Z}_{\bar{\sigma}}^{-1 / 2}$, where $\sigma, \bar{\sigma}= \pm$ are helicity labels. Using Eqs. (57), (58), and (A11), we thus obtain

$$
\left(Z_{\sigma}^{-1 / 2} X \bar{Z}_{\bar{\sigma}}^{-1 / 2}\right)_{i j}=\frac{M_{i i}^{\sigma}\left[\left(M^{\sigma}\right)^{-1} X\left(\bar{M}^{\bar{\sigma}}\right)^{-1}\right]_{i j} \bar{M}_{j j}^{\bar{\sigma}}}{\left(Z_{\sigma}^{1 / 2}\right)_{i i}\left(\bar{Z}_{\bar{\sigma}}^{1 / 2}\right)_{j j}} .
$$

On the other hand, Eqs. (71) and (72) tell us that the products $\left(Z_{\sigma}^{1 / 2}\right)_{i i}\left(\bar{Z}_{\bar{\sigma}}^{1 / 2}\right)_{i i}$ are uniquely determined, so that the same is true for $[\hat{P}(\not p)]_{i i}$. By the same token, there are no ambiguities for $i \rightarrow i$ transitions in quantum field theories in which fermion number is conserved, because each appearance of $\left(Z_{+}^{1 / 2}\right)_{k i}$ or $\left(Z_{-}^{1 / 2}\right)_{k i}$ is then always saturated by a factor of $\left(\bar{Z}_{+}^{1 / 2}\right)_{i j}$ or $\left(\bar{Z}_{-}^{1 / 2}\right)_{i j}$.

\section{WFR BIFURCATION}

Let us assume temporarily that all the Dirac fermions are stable, with $\Gamma_{i}=0$ in Eq. (14). In the complex $p^{2}$ plane, their mass shells $p^{2}=m_{i}^{2}$ are then all located on the real axis below the thresholds of $\left[A_{ \pm}\left(p^{2}\right)\right]_{i j}$ and $\left[B_{ \pm}\left(p^{2}\right)\right]_{i j}$, where the absorptive parts of the latter vanish. Then, up to a sign flip in the $i \epsilon$ prescription, which is irrelevant at this stage, the bare propagator matrix satisfies the pseudoHermiticity condition $\gamma^{0}[P(\not p)]^{\dagger} \gamma^{0}=P(\not p) \quad[6],{ }^{2}$ which implies via Eq. (5) that $\gamma^{0}[\Sigma(p)]^{\dagger} \gamma^{0}=\Sigma(\not p)$ [25] and via Eq. (6) that $A_{ \pm}^{\dagger}\left(p^{2}\right)=A_{\mp}\left(p^{2}\right)$ and $B_{ \pm}^{\dagger}\left(p^{2}\right)=B_{ \pm}\left(p^{2}\right)$ [15]. Equations (7), (10), (16), (60), and (66) then tell us that

$$
\begin{aligned}
S_{ \pm}^{\dagger}\left(p^{2}\right) & =S_{ \pm}\left(p^{2}\right), & & T_{ \pm}^{\dagger}\left(p^{2}\right)=T_{\mp}\left(p^{2}\right), \\
F_{ \pm}^{\dagger}\left(p^{2}\right) & =E_{ \pm}\left(p^{2}\right), & & \left(M^{ \pm}\right)^{\dagger}=\bar{M}^{ \pm}, \\
{\left[s_{i}^{ \pm}\left(p^{2}\right)\right]^{*} } & =s_{i}^{ \pm}\left(p^{2}\right), & & {\left[t_{i}^{ \pm}\left(p^{2}\right)\right]^{*}=t_{i}^{\mp}\left(p^{2}\right), } \\
{\left[f_{i}\left(p^{2}\right)\right]^{*} } & =f_{i}\left(p^{2}\right), & &
\end{aligned}
$$

where we have used the third equality of Eq. (A2) in the fourth equality. From the last equality in Eq. (76) in combination with Eq. (65), we conclude that $M_{i}^{*}=M_{i}$ and thus recover from Eq. (14) our assumption $\Gamma_{i}=0$, which reassures us of the self-consistency of our analysis.

\footnotetext{
${ }^{2}$ On the right-hand side of this equation, we omitted the additional term $i \int d^{4} x e^{i p \cdot x}\left\langle 0\left|\left[\Psi^{0}(x), \bar{\Psi}^{0}(0)\right]\right| 0\right\rangle$. In the noninteracting theory, its matrix elements in generation space, $\delta_{i j}\left(\frac{1}{\not p-m_{i}^{0}-i \epsilon}-\frac{1}{\not p-m_{i}^{0}+i \epsilon}\right)$, just flip the sign of the $i \epsilon$ term in $[P(\not p)]_{i j}=\frac{\delta_{i j}}{\not p-m_{i}^{0}+i \epsilon}$.
} 
From Eq. (76), it follows that the right-hand side of Eq. (71) is real and that complex conjugation of the right-hand side of Eq. (72) entails a flip of the alternating-sign labels. Furthermore, Hermitian conjugation of Eq. (57) yields

$$
\left(Z_{ \pm}^{\dagger 1 / 2}\right)_{i j}=\left(Z_{ \pm}^{\dagger 1 / 2}\right)_{i i} \frac{\bar{M}_{i j}^{ \pm}}{\bar{M}_{i i}^{ \pm}}
$$

which is to be compared with Eq. (58). It is, therefore, consistent to identify $\bar{Z}_{ \pm}^{1 / 2}=Z_{ \pm}^{\dagger 1 / 2}$, which, in the language of Eq. (20), may be written as

$$
\bar{Z}^{1 / 2}=\gamma^{0} Z^{\dagger 1 / 2} \gamma^{0} .
$$

For each value of $i$, Eqs. (71) and (72) then provide four real equations for the four real unknowns $\operatorname{Re}\left(Z_{ \pm}^{1 / 2}\right)_{i i}$ and $\operatorname{Im}\left(Z_{ \pm}^{1 / 2}\right)_{i i}$. Because of Eq. (65), however, one of these equations is redundant. In fact, the fourth of the equalities in Eqs. (71) and (72) already follows from the first three, as $\left(Z_{+}^{1 / 2}\right)_{i i}^{*}\left(Z_{-}^{1 / 2}\right)_{i i}=\left|\left(Z_{+}^{1 / 2}\right)_{i i}\right|^{2}\left|\left(Z_{-}^{1 / 2}\right)_{i i}\right|^{2} /$ $\left[\left(Z_{-}^{1 / 2}\right)_{i i}^{*}\left(Z_{+}^{1 / 2}\right)_{i i}\right]$. Again, this residual freedom does not affect Eq. (31). We may exhaust it by choosing e.g. $\operatorname{Im}\left(Z_{+}^{1 / 2}\right)_{i i}=0$, i.e. $\left(Z_{+}^{1 / 2}\right)_{i i}^{*}=\left(Z_{+}^{1 / 2}\right)_{i i}$, as was done in Ref. [15] at one loop. We shall return to this point in the paragraph before the last of Sec. IX.

We now return to the general case of unstable Dirac fermions, with $\Gamma_{i}>0$ in Eq. (14). In general, we then have $\gamma^{0}[\Sigma(\not p)]^{\dagger} \gamma^{0} \neq \Sigma(\not p)$, so that Eq. (76) no longer holds true, and the right-hand sides of Eqs. (71) and (72) loose the special complex-conjugation properties described above. This enforces the departure from Eq. (78), as anticipated in Eq. (21), which was originally observed in Ref. [25] on the basis of a one-loop analysis. We call this phenomenon WFR bifurcation [23].

\section{PROOF OF UNIT-RESIDUE PROPERTY}

We now study the limit $p^{2} \rightarrow M_{n}^{2}$, in which the Dirac fermion $n$ goes on its mass shell, and explicitly demonstrate that, owing to the on-shell WFR conditions of Eqs. (33)-(36), the resonating diagonal element $[\hat{P}(\not p)]_{n n}$ of the renormalized propagator in Eq. (28) has unit residue, in compliance with the LSZ reduction formalism [22]. A similar proof was presented in Ref. [21] for the case of stability.

Let us consider the coefficient matrices in the first and second lines of Eq. (28),

$$
\hat{P}_{\mp}(\not p)=\left[\not p+\hat{D}_{ \pm}\left(p^{2}\right)\right] \hat{S}_{ \pm}^{-1}\left(p^{2}\right) \frac{\operatorname{adj}\left[p^{2}-\hat{E}_{ \pm}\left(p^{2}\right)\right]}{\operatorname{det}\left[p^{2}-\hat{E}_{ \pm}\left(p^{2}\right)\right.},
$$

where we have rewritten the last factor using Eq. (A7). Taylor expanding the denominator in Eq. (79) about $p^{2}=M_{n}^{2}$ with the aid of Eq. (A12), we obtain

$$
\begin{aligned}
\operatorname{det}[ & \left.p^{2}-\hat{E}_{ \pm}\left(p^{2}\right)\right] \\
= & \left(p^{2}-M_{n}^{2}\right) \operatorname{tr}\left\{\operatorname{adj}\left[M_{n}^{2}-\hat{E}_{ \pm}\left(M_{n}^{2}\right)\right]\left[I_{N}-\hat{E}_{ \pm}^{\prime}\left(M_{n}^{2}\right)\right]\right\} \\
& +\mathcal{O}\left[\left(p^{2}-M_{n}^{2}\right)^{2}\right],
\end{aligned}
$$

where we have exploited the fact that the $p^{2}$-independent term vanishes according to Eqs. (13) and (29). Next, we conclude from Eqs. (46) and (45) that $\left[M_{n}^{2}-\hat{E}_{ \pm}\left(M_{n}^{2}\right)\right]_{i j}=0$ if $i=n$ and that $\left[M_{n}^{2}-\hat{F}_{ \pm}\left(M_{n}^{2}\right)\right]_{i j}=0$ if $j=n$, so that the adjugates of these matrices take the forms

$$
\begin{aligned}
& \left\{\operatorname{adj}\left[M_{n}^{2}-\hat{E}_{ \pm}\left(M_{n}^{2}\right)\right]\right\}_{i j}=x_{i n}^{ \pm} \delta_{n j}, \\
& \left\{\operatorname{adj}\left[M_{n}^{2}-\hat{F}_{ \pm}\left(M_{n}^{2}\right)\right]\right\}_{i j}=\delta_{i n} y_{n j}^{ \pm},
\end{aligned}
$$

where $x_{i n}^{ \pm}$and $y_{n j}^{ \pm}$are the entries of the $n$th column of $\operatorname{adj}\left[M_{n}^{2}-\hat{E}_{ \pm}\left(M_{n}^{2}\right)\right]$ and the $n$th row of $\operatorname{adj}\left[M_{n}^{2}-\hat{F}_{ \pm}\left(M_{n}^{2}\right)\right]$, respectively, while all other entries vanish. Furthermore, with the help of Eq. (A10), we derive from Eq. (27) the relationship

$$
\hat{S}_{ \pm}^{-1}\left(p^{2}\right) \operatorname{adj}\left[p^{2}-\hat{E}_{ \pm}\left(p^{2}\right)\right] \hat{S}_{ \pm}\left(p^{2}\right)=\operatorname{adj}\left[p^{2}-\hat{F}_{ \pm}\left(p^{2}\right)\right],
$$

and hence

$$
\hat{S}_{ \pm}^{-1}\left(p^{2}\right) \operatorname{adj}\left[p^{2}-\hat{E}_{ \pm}\left(p^{2}\right)\right]=\operatorname{adj}\left[p^{2}-\hat{F}_{ \pm}\left(p^{2}\right)\right] \hat{S}_{ \pm}^{-1}\left(p^{2}\right)
$$

Putting $p^{2}=M_{n}^{2}$ and inserting Eq. (81) into Eq. (83), we get

$$
\begin{aligned}
& \left\{\hat{S}_{ \pm}^{-1}\left(M_{n}^{2}\right) \operatorname{adj}\left[M_{n}^{2}-\hat{E}_{ \pm}\left(M_{n}^{2}\right)\right]\right\}_{i j}=X_{i n}^{ \pm} \delta_{n j} \\
& \quad=\left\{\operatorname{adj}\left[M_{n}^{2}-\hat{F}_{ \pm}\left(M_{n}^{2}\right)\right] \hat{S}_{ \pm}^{-1}\left(M_{n}^{2}\right)\right\}_{i j}=\delta_{i n} Y_{n j}^{ \pm}
\end{aligned}
$$

where

$$
X_{i n}^{ \pm}=\sum_{k=1}^{N}\left[\hat{S}_{ \pm}^{-1}\left(M_{n}^{2}\right)\right]_{i k} x_{k n}^{ \pm}, \quad Y_{n j}^{ \pm}=\sum_{k=1}^{N} y_{n k}^{ \pm}\left[\hat{S}_{ \pm}^{-1}\left(M_{n}^{2}\right)\right]_{k j} .
$$

Equation (84) tells us that all the quantities $X_{i n}^{ \pm}$and $Y_{n j}^{ \pm}$ vanish, except for $Z_{n}^{ \pm}=X_{n n}^{ \pm}=Y_{n n}^{ \pm}$, so that

$$
\left\{\hat{S}_{ \pm}^{-1}\left(M_{n}^{2}\right) \operatorname{adj}\left[M_{n}^{2}-\hat{E}_{ \pm}\left(M_{n}^{2}\right)\right]\right\}_{i j}=\delta_{i n} Z_{n}^{ \pm} \delta_{n j} .
$$

The product in Eq. (86) appears in the numerator of Eq. (79) in the limit $p^{2} \rightarrow M_{n}^{2}$. We may exploit Eq. (86) once more by rewriting the trace in Eq. (80) as

$$
\begin{aligned}
& \operatorname{tr}\left\{\operatorname{adj}\left[M_{n}^{2}-\hat{E}_{ \pm}\left(M_{n}^{2}\right)\right]\left[I_{N}-\hat{E}_{ \pm}^{\prime}\left(M_{n}^{2}\right)\right]\right\} \\
& =\operatorname{tr}\left\{\hat{S}_{ \pm}^{-1}\left(M_{n}^{2}\right) \operatorname{adj}\left[M_{n}^{2}-\hat{E}_{ \pm}\left(M_{n}^{2}\right)\right]\left[I_{N}-\hat{E}_{ \pm}^{\prime}\left(M_{n}^{2}\right)\right] \hat{S}_{ \pm}\left(M_{n}^{2}\right)\right\} \\
& =Z_{n}^{ \pm}\left\{\left[I_{N}-\hat{E}_{ \pm}^{\prime}\left(M_{n}^{2}\right)\right] \hat{S}_{ \pm}\left(M_{n}^{2}\right)\right\}_{n n} .
\end{aligned}
$$


With the help of Eq. (A17), we derive from Eq. (27) the relationship

$$
\begin{aligned}
{\left[I_{N}-\right.} & \left.\hat{E}_{ \pm}^{\prime}\left(p^{2}\right)\right] \hat{S}_{ \pm}\left(p^{2}\right) \\
= & \hat{S}_{ \pm}\left(p^{2}\right)-\hat{T}_{\mp}^{\prime}\left(p^{2}\right) \hat{D}_{ \pm}\left(p^{2}\right)+\hat{C}_{ \pm}\left(p^{2}\right) \hat{S}_{\mp}^{\prime}\left(p^{2}\right) \hat{D}_{ \pm}\left(p^{2}\right) \\
& -\hat{C}_{ \pm}\left(p^{2}\right) \hat{T}_{ \pm}^{\prime}\left(p^{2}\right)+\hat{E}_{ \pm}\left(p^{2}\right) \hat{S}_{ \pm}^{\prime}\left(p^{2}\right) .
\end{aligned}
$$

Putting $p^{2}=M_{n}^{2}$ in the matrix element with $i=j=n$ of Eq. (88) and inserting Eqs. (43), (44), and (46), we get

$$
\begin{aligned}
\left\{\left[I_{N}-\hat{E}_{ \pm}^{\prime}\left(M_{n}^{2}\right)\right] \hat{S}_{ \pm}\left(M_{n}^{2}\right)\right\}_{n n} & \\
= & {\left[\hat{S}_{ \pm}\left(M_{n}^{2}\right)\right]_{n n}+M_{n}^{2}\left\{\left[\hat{S}_{+}^{\prime}\left(M_{n}^{2}\right)\right]_{n n}+\left[\hat{S}_{-}^{\prime}\left(M_{n}^{2}\right)\right]_{n n}\right\} } \\
& \quad-M_{n}\left\{\left[\hat{T}_{+}^{\prime}\left(M_{n}^{2}\right)\right]_{n n}+\left[\hat{T}_{-}^{\prime}\left(M_{n}^{2}\right)\right]_{n n}\right\}=1
\end{aligned}
$$

where we have used Eqs. (40) and (41) in the last step. Inserting Eq. (89) into Eq. (87), we obtain

$$
\operatorname{tr}\left\{\operatorname{adj}\left[M_{n}^{2}-\hat{E}_{ \pm}\left(M_{n}^{2}\right)\right]\left[I_{N}-\hat{E}_{ \pm}^{\prime}\left(M_{n}^{2}\right)\right]\right\}=Z_{n}^{ \pm} .
$$

Taking the limit $p^{2} \rightarrow M_{n}^{2}$ and inserting Eqs. (80), (86), and (90) into Eq. (79), we have

$$
\begin{aligned}
{\left[\hat{P}_{\mp}(\not p)\right]_{i j} } & =\frac{\sum_{k=1}^{N}\left[\not p+\hat{D}_{ \pm}\left(M_{n}^{2}\right)\right]_{i k} \delta_{k n} Z_{n}^{ \pm} \delta_{n j}+\mathcal{O}\left(p^{2}-M_{n}^{2}\right)}{\left(p^{2}-M_{n}^{2}\right) Z_{n}^{ \pm}+\mathcal{O}\left[\left(p^{2}-M_{n}^{2}\right)^{2}\right]} \\
& =\frac{\delta_{i n} \delta_{n j}}{\not p-M_{n}}+\mathcal{O}(1),
\end{aligned}
$$

where we have used Eq. (43) in the second equality. Finally, inserting Eq. (91) into the first and second lines of Eq. (28) and using $a_{+}+a_{-}=I_{4}$, we recover Eq. (31) in the limit $p^{2} \rightarrow M_{n}^{2}$, which was to be demonstrated. Alternatively, Eq. (31) may be derived from the third and fourth lines in Eq. (28).

\section{UNSTABLE DIRAC FERMION TO ALL ORDERS}

We now consider the important special case of a single unstable Dirac fermion, in which the general expressions derived in Secs. II-V significantly simplify. Then there are just four Lorentz-invariant functions in Eq. (7),

$$
S_{ \pm}\left(p^{2}\right)=1-B_{ \pm}\left(p^{2}\right), \quad T_{ \pm}\left(p^{2}\right)=m^{0}+A_{ \pm}\left(p^{2}\right),
$$

the expressions in the second line of Eq. (10) all coincide,

$$
f\left(p^{2}\right)=E_{ \pm}\left(p^{2}\right)=F_{ \pm}\left(p^{2}\right)=\frac{T_{+}\left(p^{2}\right) T_{-}\left(p^{2}\right)}{S_{+}\left(p^{2}\right) S_{-}\left(p^{2}\right)},
$$

and, according to Eq. (13), the pole position is defined by

$$
M^{2}=f\left(M^{2}\right) .
$$

According to Eq. (25), the renormalized propagator reads

$$
\begin{aligned}
\hat{P}(\not p)= & {\left[Z_{-}^{-1 / 2} \not p+Z_{+}^{-1 / 2} \frac{T_{-}\left(p^{2}\right)}{S_{+}\left(p^{2}\right)}\right] } \\
& \times \frac{1}{S_{-}\left(p^{2}\right)} \frac{1}{p^{2}-f\left(p^{2}\right)} \bar{Z}_{-}^{-1 / 2} a_{+}+(+\leftrightarrow-) .
\end{aligned}
$$

According to Eqs. (42) and (40), the four WFR constants are fixed by

$$
\begin{aligned}
& M \hat{S}_{+}\left(M^{2}\right)=M \hat{S}_{-}\left(M^{2}\right)=\hat{T}_{+}\left(M^{2}\right)=\hat{T}_{-}\left(M^{2}\right), \\
& \hat{S}_{+}\left(M^{2}\right)+M^{2}\left[\hat{S}_{+}^{\prime}\left(M^{2}\right)+\hat{S}_{-}^{\prime}\left(M^{2}\right)\right] \\
& \quad-M\left[\hat{T}_{+}^{\prime}\left(M^{2}\right)+\hat{T}_{-}^{\prime}\left(M^{2}\right)\right]=1,
\end{aligned}
$$

respectively, yielding

$$
\begin{aligned}
& Z_{ \pm}^{-1 / 2} \bar{Z}_{ \pm}^{-1 / 2}=S_{ \pm}\left(M^{2}\right)\left[1-f^{\prime}\left(M^{2}\right)\right], \\
& Z_{ \pm}^{-1 / 2} \bar{Z}_{\mp}^{-1 / 2}=\frac{T_{ \pm}\left(M^{2}\right)}{M}\left[1-f^{\prime}\left(M^{2}\right)\right],
\end{aligned}
$$

in analogy to Eqs. (71) and (72), respectively. The renormalized propagator is thus uniquely determined and may be written in the compact form

$$
\begin{aligned}
\hat{P}(\not p)= & {\left[\not p+M \frac{S_{+}\left(M^{2}\right)}{S_{+}\left(p^{2}\right)} \frac{T_{-}\left(p^{2}\right)}{T_{-}\left(M^{2}\right)}\right] } \\
& \times \frac{S_{-}\left(M^{2}\right)}{S_{-}\left(p^{2}\right)} \frac{1-f^{\prime}\left(M^{2}\right)}{p^{2}-f\left(p^{2}\right)} a_{+}+(+\leftrightarrow-),
\end{aligned}
$$

which evidently has unit residue at the physical pole $p=M$. We may impose one more condition, e.g. $\bar{Z}_{+}^{1 / 2}=$ $Z_{+}^{1 / 2}$ or $\bar{Z}_{-}^{1 / 2}=Z_{-}^{1 / 2}$, without spoiling the unit-residue property, but not both, as was illustrated in Ref. [28].

\section{MIXED SYSTEM OF UNSTABLE DIRAC FERMIONS AT ONE LOOP}

In order to explore the anatomy of the all-order expressions for the renormalization constants $\delta M_{i},\left(Z_{ \pm}^{1 / 2}\right)_{i j}$, and $\left(\bar{Z}_{ \pm}^{1 / 2}\right)_{i j}$ derived in Secs. II-V, it is useful to consider a mixed system of $N$ unstable Dirac fermions at one loop. We thus assume the self-energy functions $\left[A_{ \pm}\left(p^{2}\right)\right]_{i j}$ and $\left[B_{ \pm}\left(p^{2}\right)\right]_{i j}$ to be known through $\mathcal{O}(\alpha)$. Our goal is to express $\delta M_{i},\left(Z_{ \pm}^{1 / 2}\right)_{i j}$, and $\left(\bar{Z}_{ \pm}^{1 / 2}\right)_{i j}$ in terms of $\left[A_{ \pm}\left(p^{2}\right)\right]_{i j}$ and $\left[B_{ \pm}\left(p^{2}\right)\right]_{i j}$ through $\mathcal{O}(\alpha)$. We may then check the outcome against the literature $[15,25]$.

Expanding Eq. (10) through $\mathcal{O}(\alpha)$, we obtain

$$
\begin{aligned}
& {\left[E_{ \pm}\left(p^{2}\right)\right]_{i j}=\left(m_{i}^{0}\right)^{2} \delta_{i j}+\left[G_{ \pm}\left(p^{2}\right)\right]_{i j}+\mathcal{O}\left(\alpha^{2}\right),} \\
& {\left[F_{ \pm}\left(p^{2}\right)\right]_{i j}=\delta_{i j}\left(m_{j}^{0}\right)^{2}+\left[H_{ \pm}\left(p^{2}\right)\right]_{i j}+\mathcal{O}\left(\alpha^{2}\right),}
\end{aligned}
$$

with 


$$
\begin{aligned}
{\left[G_{ \pm}\left(p^{2}\right)\right]_{i j}=} & M_{i}\left[A_{ \pm}\left(p^{2}\right)\right]_{i j}+\left[A_{\mp}\left(p^{2}\right)\right]_{i j} M_{j} \\
& +M_{i}^{2}\left[B_{ \pm}\left(p^{2}\right)\right]_{i j}+M_{i}\left[B_{\mp}\left(p^{2}\right)\right]_{i j} M_{j}, \\
{\left[H_{ \pm}\left(p^{2}\right)\right]_{i j}=} & M_{i}\left[A_{ \pm}\left(p^{2}\right)\right]_{i j}+\left[A_{\mp}\left(p^{2}\right)\right]_{i j} M_{j} \\
& +\left[B_{ \pm}\left(p^{2}\right)\right]_{i j} M_{j}^{2}+M_{i}\left[B_{\mp}\left(p^{2}\right)\right]_{i j} M_{j},
\end{aligned}
$$

where we have used Eq. (18) to replace $m_{i}^{0}$ by $M_{i}$ at $\mathcal{O}(\alpha)$. Observing that the diagonal elements in Eq. (100) coincide, we define

$$
\begin{aligned}
I_{i}\left(p^{2}\right)= & {\left[G_{+}\left(p^{2}\right)\right]_{i i}=\left[G_{-}\left(p^{2}\right)\right]_{i i}=\left[H_{+}\left(p^{2}\right)\right]_{i i}=\left[H_{-}\left(p^{2}\right)\right]_{i i} } \\
= & M_{i}\left\{\left[A_{+}\left(p^{2}\right)\right]_{i i}+\left[A_{-}\left(p^{2}\right)\right]_{i i}\right\}+M_{i}^{2}\left\{\left[B_{+}\left(p^{2}\right)\right]_{i i}\right. \\
& \left.+\left[B_{-}\left(p^{2}\right)\right]_{i i}\right\} .
\end{aligned}
$$

For definiteness, we assume that $N=3$ in the following. Later on, the general case may be recovered without further ado. Without loss of generality, we take $p^{2}=M_{1}^{2}$. Then, Eq. (16) becomes

$$
\begin{aligned}
M_{i 1}^{ \pm}= & \left\{\operatorname{adj}\left[M_{1}^{2}-F_{ \pm}\left(M_{1}^{2}\right)\right]\right\}_{i 1} \\
= & \left(M_{1}^{2}-M_{2}^{2}\right)\left(M_{1}^{2}-M_{3}^{2}\right)\left(1-X_{1}, \frac{H_{21}^{ \pm}}{M_{1}^{2}-M_{2}^{2}}, \frac{H_{31}^{ \pm}}{M_{1}^{2}-M_{3}^{2}}\right)^{T} \\
& +\mathcal{O}\left(\alpha^{2}\right), \\
\bar{M}_{1 j}^{ \pm}= & \left\{\operatorname{adj}\left[M_{1}^{2}-E_{ \pm}\left(M_{1}^{2}\right)\right]\right\}_{1 j} \\
= & \left(M_{1}^{2}-M_{2}^{2}\right)\left(M_{1}^{2}-M_{3}^{2}\right)\left(1-X_{1}, \frac{G_{12}^{ \pm}}{M_{1}^{2}-M_{2}^{2}}, \frac{G_{13}^{ \pm}}{M_{1}^{2}-M_{3}^{2}}\right) \\
& +\mathcal{O}\left(\alpha^{2}\right),
\end{aligned}
$$

where

$$
\begin{aligned}
& X_{1}=\frac{2 M_{2} \delta M_{2}+I_{2}\left(M_{1}^{2}\right)}{M_{1}^{2}-M_{2}^{2}}+\frac{2 M_{3} \delta M_{3}+I_{3}\left(M_{1}^{2}\right)}{M_{1}^{2}-M_{3}^{2}}, \\
& H_{i 1}^{ \pm}=\left[H_{ \pm}\left(M_{1}^{2}\right)\right]_{i 1}, \quad G_{1 j}^{ \pm}=\left[G_{ \pm}\left(M_{1}^{2}\right)\right]_{1 j} .
\end{aligned}
$$

Thus, Eq. (60) becomes

$$
\begin{aligned}
s_{1}^{ \pm}\left(p^{2}\right)= & {\left[\bar{M}^{ \pm} S_{ \pm}\left(p^{2}\right) M^{ \pm}\right]_{11} } \\
= & \left(M_{1}^{2}-M_{2}^{2}\right)^{2}\left(M_{1}^{2}-M_{3}^{2}\right)^{2}\left\{1-2 X_{1}-\left[B_{ \pm}\left(p^{2}\right)\right]_{11}\right\} \\
& +\mathcal{O}\left(\alpha^{2}\right), \\
t_{1}^{ \pm}\left(p^{2}\right)= & {\left[\bar{M}^{\mp} T_{ \pm}\left(p^{2}\right) M^{ \pm}\right]_{11} } \\
= & \left(M_{1}^{2}-M_{2}^{2}\right)^{2}\left(M_{1}^{2}-M_{3}^{2}\right)^{2}\left\{m_{1}^{0}\left(1-2 X_{1}\right)\right. \\
& \left.+\left[A_{ \pm}\left(p^{2}\right)\right]_{11}\right\}+\mathcal{O}\left(\alpha^{2}\right) .
\end{aligned}
$$

Consequently, Eq. (66) becomes

$$
\begin{aligned}
f_{1}\left(p^{2}\right) & =\frac{t_{1}^{+}\left(p^{2}\right) t_{1}^{-}\left(p^{2}\right)}{s_{1}^{+}\left(p^{2}\right) s_{1}^{-}\left(p^{2}\right)} \\
& =\left(m_{1}^{0}\right)^{2}+I_{1}\left(p^{2}\right)+\mathcal{O}\left(\alpha^{2}\right) .
\end{aligned}
$$

Using Eq. (67), we hence find the mass counterterm to be

$$
\begin{aligned}
\delta M_{1} & =m_{1}^{0}-\sqrt{f_{1}\left(M_{1}^{2}\right)} \\
& =-\frac{I_{1}\left(M_{1}^{2}\right)}{2 M_{1}}+\mathcal{O}\left(\alpha^{2}\right) .
\end{aligned}
$$

Inserting Eqs. (102) and (104) into Eqs. (71) and (72), we get

$$
\begin{aligned}
\left(\bar{Z}_{ \pm}^{1 / 2}\right)_{11}\left(Z_{ \pm}^{1 / 2}\right)_{11}= & \frac{\bar{M}_{11}^{ \pm} M_{11}^{ \pm}}{s_{1}^{ \pm}\left[1-f_{1}^{\prime}\left(M_{1}^{2}\right)\right]} \\
= & 1+\left[B_{ \pm}\left(M_{1}^{2}\right)\right]_{11}+f_{1}^{\prime}\left(M_{1}^{2}\right)+\mathcal{O}\left(\alpha^{2}\right) \\
\left(\bar{Z}_{\mp}^{1 / 2}\right)_{11}\left(Z_{ \pm}^{1 / 2}\right)_{11}= & \frac{M_{1} \bar{M}_{11}^{\mp} M_{11}^{ \pm}}{t_{1}^{ \pm}\left[1-f_{1}^{\prime}\left(M_{1}^{2}\right)\right]} \\
= & 1-\frac{\delta M_{1}+\left[A_{ \pm}\left(M_{1}^{2}\right)\right]_{11}}{M_{1}} \\
& +f_{1}^{\prime}\left(M_{1}^{2}\right)+\mathcal{O}\left(\alpha^{2}\right)
\end{aligned}
$$

where

$$
\begin{aligned}
f_{1}^{\prime}\left(p^{2}\right)= & I_{1}^{\prime}\left(p^{2}\right)+\mathcal{O}\left(\alpha^{2}\right) \\
= & M_{1}\left\{\left[A_{+}^{\prime}\left(p^{2}\right)\right]_{11}+\left[A_{-}^{\prime}\left(p^{2}\right)\right]_{11}\right\} \\
& +M_{1}^{2}\left\{\left[B_{+}^{\prime}\left(p^{2}\right)\right]_{11}+\left[B_{-}^{\prime}\left(p^{2}\right)\right]_{11}\right\}+\mathcal{O}\left(\alpha^{2}\right) .
\end{aligned}
$$

Finally, substituting Eq. (102) into Eqs. (57) and (58), and observing that $H_{i 1}^{ \pm}$with $i \neq 1$ and $G_{1 j}^{ \pm}$with $j \neq 1$ are already of $\mathcal{O}(\alpha)$, we obtain

$$
\begin{aligned}
\left(Z_{ \pm}^{1 / 2}\right)_{i 1} & =\frac{M_{i 1}^{ \pm}}{M_{11}^{ \pm}}\left(Z_{ \pm}^{1 / 2}\right)_{11} \\
& =\frac{H_{i 1}^{ \pm}}{M_{1}^{2}-M_{i}^{2}}+\mathcal{O}\left(\alpha^{2}\right) \quad(i \neq 1), \\
\left(\bar{Z}_{ \pm}^{1 / 2}\right)_{1 j} & =\left(\bar{Z}_{ \pm}^{1 / 2}\right)_{11} \frac{\bar{M}_{1 j}^{ \pm}}{\bar{M}_{11}^{ \pm}} \\
& =\frac{G_{1 j}^{ \pm}}{M_{1}^{2}-M_{j}^{2}}+\mathcal{O}\left(\alpha^{2}\right) \quad(j \neq 1) .
\end{aligned}
$$

The counterparts of Eqs. (106), (107), and (109) for $p^{2}=M_{2}^{2}$ and $p^{2}=M_{3}^{2}$ follow by cyclic permutations, and the generalization to arbitrary values of $N$ is straightforward. Expanding

$Z_{ \pm}^{1 / 2}=\left(I_{N}+\delta Z_{ \pm}\right)^{1 / 2}=I_{N}+1 / 2 \delta Z_{ \pm}+\mathcal{O}\left(\alpha^{2}\right)$,

$\bar{Z}_{ \pm}^{1 / 2}=\left(I_{N}+\delta \bar{Z}_{ \pm}\right)^{1 / 2}=I_{N}+1 / 2 \delta \bar{Z}_{ \pm}+\mathcal{O}\left(\alpha^{2}\right)$,

we have

$$
\delta M_{i}=-\frac{I_{i}\left(M_{i}^{2}\right)}{2 M_{i}}+\mathcal{O}\left(\alpha^{2}\right),
$$




$$
\begin{gathered}
\frac{1}{2}\left[\left(\delta \bar{Z}_{ \pm}\right)_{i i}+\left(\delta Z_{ \pm}\right)_{i i}\right]=\left[B_{ \pm}\left(M_{i}^{2}\right)\right]_{i i}+I_{i}^{\prime}\left(M_{i}^{2}\right)+\mathcal{O}\left(\alpha^{2}\right) \\
\frac{1}{2}\left[\left(\delta \bar{Z}_{\mp}\right)_{i i}+\left(\delta Z_{ \pm}\right)_{i i}\right] \\
=\frac{I_{i}\left(M_{i}^{2}\right)}{2 M_{i}^{2}}-\frac{\left[A_{ \pm}\left(M_{i}^{2}\right)\right]_{i i}}{M_{i}}+I_{i}^{\prime}\left(M_{i}^{2}\right)+\mathcal{O}\left(\alpha^{2}\right), \\
\frac{1}{2}\left(\delta Z_{ \pm}\right)_{i j}=\frac{\left[H_{ \pm}\left(M_{j}^{2}\right)\right]_{i j}}{M_{j}^{2}-M_{i}^{2}}+\mathcal{O}\left(\alpha^{2}\right) \quad(i \neq j) \\
\frac{1}{2}\left(\delta \bar{Z}_{ \pm}\right)_{i j}=\frac{\left[G_{ \pm}\left(M_{i}^{2}\right)\right]_{i j}}{M_{i}^{2}-M_{j}^{2}}+\mathcal{O}\left(\alpha^{2}\right) \quad(i \neq j) .
\end{gathered}
$$

Following Eq. (74), we may put, as in Ref. [15],

$$
\left(\delta \bar{Z}_{+}\right)_{i i}=\left(\delta Z_{+}\right)_{i i} .
$$

Then, all diagonal WFR matrix elements are uniquely fixed by Eqs. (112) and (113) as

$$
\begin{aligned}
&\left(\delta \bar{Z}_{+}\right)_{i i}=\left(\delta Z_{+}\right)_{i i}=\left[B_{+}\left(M_{i}^{2}\right)\right]_{i i}+I_{i}^{\prime}\left(M_{i}^{2}\right)+\mathcal{O}\left(\alpha^{2}\right), \\
&\left(\delta Z_{-}\right)_{i i}=\frac{\left[A_{+}\left(M_{i}^{2}\right)\right]_{i i}-\left[A_{-}\left(M_{i}^{2}\right)\right]_{i i}}{M_{i}} \\
&+\left[B_{-}\left(M_{i}^{2}\right)\right]_{i i}+I_{i}^{\prime}\left(M_{i}^{2}\right)+\mathcal{O}\left(\alpha^{2}\right), \\
&\left(\delta \bar{Z}_{-}\right)_{i i}= \\
&+\left[A_{-}\left(M_{i}^{2}\right)\right]_{i i}-\left[A_{+}\left(M_{i}^{2}\right)\right]_{i i} \\
& M_{i} \\
&\left.+B_{-}\left(M_{i}^{2}\right)\right]_{i i}+I_{i}^{\prime}\left(M_{i}^{2}\right)+\mathcal{O}\left(\alpha^{2}\right) .
\end{aligned}
$$

Equations (111), (114), (117), and (118) agree with Eqs. (3.17), (3.13), (3.16), and (3.15) in Ref. [15], respectively. In the spirit of Ref. [15], Eqs. (115) and (119) correspond to the Hermitian conjugates of Eqs. (3.13) and (3.15), respectively. Furthermore, Eqs. (111), (114), (115), and the combination of Eqs. (112) and (113) coincide with Eqs. (4.5), (3.3), (3.4), and (4.3) in Ref. [25], respectively.

As for the case of stability at two loops, Eqs. (17) and (65) are found to coincide with Eq. (23) of Ref. [21], which was derived there by solving one of the secular equations given in Eq. (13). Explicit two-loop expressions for the building blocks of Eqs. (57), (58), (65), (71), and (72) may be found in Eqs. (40) and (41) of Ref. [23].

\section{CONCLUSIONS}

We renormalized the propagator matrix of a mixed system of unstable Dirac fermions in a general paritynonconserving quantum field theory adopting the pole scheme, in which the pole masses serve as the renormalized masses. The squares of the pole masses are the complex poles of the propagator matrix. The inverse propagator matrix is built up by the one-particle-irreducible Feynman diagrams pertaining to the transitions of fermion $j$ to fermion $i$ order by order in perturbation theory. In gauge theories, the pole masses are expected to be gauge independent. This was proven for the SM [19] using Nielsen identities [20]. In spontaneously broken gauge theories, one needs to include the tadpoles to ensure the gauge independence of the mass counterterms [5,29,30]. This then carries over to the pole masses because the bare masses are gauge independent as a matter of principle.

The WFR matrices were determined by requiring that each diagonal element of the renormalized propagator matrix has unit residue if the respective fermion is on its mass shell. This renormalization condition is singled out by the LSZ reduction formalism [22] because it avoids finite renormalizations that are otherwise required. In this sense, it may be considered scheme independent. While the products of WFR matrix elements that appear on the diagonal of the renormalized propagator matrix are uniquely determined in this way, there is some residual freedom in fixing such factors for the nondiagonal entries. We proposed an additional WFR condition, in Eq. (74), to exhaust this freedom. In the case of instability, we encountered WFR bifurcation, i.e. the WFR matrices of the in and out states are no longer related by Hermitian conjugation, as indicated in Eq. (21).

The dressed propagator matrix and the renormalization constants are expressed in terms of the unrenormalized self-energies of the $j \rightarrow i$ transitions, which have scalar, pseudoscalar, vector, and axial-vector parts. We presented closed analytic results, which are valid to all orders because we did not perform a perturbative expansion. Specifically, the dressed propagator matrix is given by Eq. (25), the pole-mass counterterms by Eq. (67), the diagonal elements of the WFR matrices by Eqs. (73) and (74), and the nondiagonal ones by Eqs. (57) and (58). In these formulas, the renormalized masses $M_{i}$ enter as arguments $p^{2}=M_{i}^{2}$ of the various self-energy functions, and it is understood that the latter are evaluated from the bare Lagrangian of the considered quantum field theory, so that the masses, couplings, and mixing angles on which they depend are all bare parameters to start with.

At one loop, the well-known results for stable [15] and unstable [25] fermions were recovered, in Eqs. (111)-(119). In the special case of a single unstable fermion, the all-order renormalized propagator was found to take a particularly simple form, as given by Eq. (98).

As already pointed out in Ref. [23], important phenomenological applications of the results presented here include (i) perturbative treatments of specific particle scattering or decay processes involving unstable Dirac fermions, (ii) iterative evaluations of total decay widths of Dirac fermions through higher orders, and (iii) changes from the pole scheme adopted here to any other scheme of mass renormalization implemented with the same method of regularization, such as the modified minimal-subtraction 
scheme [31] of dimensional regularization [32] appropriately extended from QCD to the SM [30].

The all-order renormalization of the propagaror matrix of a system of unstable Majorana fermions with intergeneration mixing is discussed elsewhere [33]. In contrast to the case of unstable Dirac fermions, the WFR matrices of the in and out states are then uniquely fixed, while they again bifurcate in the sense that they are no longer related by pseudo-Hermitian conjugation.

\section{ACKNOWLEDGMENTS}

We thank Alberto Sirlin for numerous valuable discussions leading to Ref. [21], for carefully reading this manuscript and verifying its equations, for recommending the use of the parameterization in Eq. (14), and for pointing out that the solutions in Eqs. (53) and (54) are special cases of the general ones in Eq. (51). This research was supported in part by the German Research Foundation through the Collaborative Research Center No. 676 Particles, Strings and the Early Universe-The Structure of Matter and Space Time and by the National Science Foundation under Grant No. PHY11-25915.

\section{APPENDIX A: MATRIX ALGEBRA}

In this appendix, we collect a few useful theorems of matrix algebra.

Let $A$ be a quadratic $(n \times n)$ matrix. Then the adjugate (classical adjoint) $\operatorname{adj} A=C^{T}$ of $A$ is the transpose of the matrix $C$ whose elements $C_{i j}$ are the cofactors of the elements $A_{i j}$ of $A$. We recall that the cofactor $C_{i j}$ of the element $A_{i j}$ of $A$ is $(-1)^{i+j}$ times the determinant of the $(n-1) \times$ $(n-1)$ matrix obtained by deleting the $i$ th row and the $j$ th column of $A$. Then, the following theorem holds:

$$
A \operatorname{adj} A=(\operatorname{adj} A) A=(\operatorname{det} A) I_{n} .
$$

This may be immediately understood by observing that, according to Laplace's expansion formula, $\sum_{k=1}^{n} A_{i k} C_{j k}$ is the determinant of the matrix obtained from $A$ by replacing the $j$ th row by the $i$ th row and $\sum_{k=1}^{n} C_{k i} A_{k j}$ is the determinant of the matrix obtained from $A$ by replacing the $i$ th column by the $j$ th column. If $i=j$, then, in both cases, the result is just $\operatorname{det} A$. If $i \neq j$, then it is zero because these determinants have two identical rows and columns, respectively. The properties

$\operatorname{adj}\left(A^{T}\right)=(\operatorname{adj} A)^{T}, \quad \operatorname{adj}\left(A^{*}\right)=(\operatorname{adj} A)^{*}$,

$\operatorname{adj}\left(A^{\dagger}\right)=(\operatorname{adj} A)^{\dagger}, \quad \operatorname{adj}\left(\lambda I_{n}\right)=\lambda^{n-1} I_{n}$,

where $\lambda$ is a number, follow from the definition of $\operatorname{adj} A$ with the help of $\operatorname{det}\left(A^{T}\right)=\operatorname{det} A$, $\operatorname{det}\left(A^{*}\right)=(\operatorname{det} A)^{*}=$ $\operatorname{det}\left(A^{\dagger}\right)$, and $\operatorname{det}\left(\lambda I_{n}\right)=\lambda^{n}$. If $A$ and $B$ are two quadratic matrices, then

$$
\operatorname{adj}(A B)=(\operatorname{adj} B)(\operatorname{adj} A) .
$$

In fact, using Eq. (A1) together with $\operatorname{det}(A B)=$ $(\operatorname{det} A)(\operatorname{det} B)$, we obtain $A B(\operatorname{adj} B)(\operatorname{adj} A)=A B \operatorname{adj}(A B)$ and $(\operatorname{adj} B)(\operatorname{adj} A) A B=\operatorname{adj}(A B) A B$. Substituting $B=\lambda I_{n}$ into Eq. (A3) and using the last equality in Eq. (A2), we obtain

$$
\operatorname{adj}(\lambda A)=\lambda^{n-1} \operatorname{adj} A .
$$

Taking the determinant of Eq. (A1), we obtain

$$
\operatorname{det} \operatorname{adj} A=(\operatorname{det} A)^{n-1} .
$$

Taking the adjugate of Eq. (A1), multiplying the outcome with $A$, and using Eq. (A3), the last equality in Eq. (A2), and Eq. (A1), we obtain

$$
\operatorname{adj} \operatorname{adj} A=(\operatorname{det} A)^{n-2} A .
$$

In Eqs. (A5) and (A6), we have assumed that $\operatorname{det} A \neq 0$ if $n<1$ and $n<2$, respectively.

If $\operatorname{det} A \neq 0$, then $A$ is regular, and its inverse may be evaluated using Eq. (A1), as

$$
A^{-1}=(\operatorname{det} A)^{-1} \operatorname{adj} A \text {. }
$$

Likewise, we have

$$
\operatorname{adj} A=(\operatorname{det} A) A^{-1},
$$

from which, using $\operatorname{det}\left(A^{-1}\right)=(\operatorname{det} A)^{-1}$, we obtain

$$
\operatorname{adj}\left(A^{-1}\right)=(\operatorname{det} A)^{-1} A=(\operatorname{adj} A)^{-1} .
$$

Let the two quadratic matrices $A$ and $B$ be similar, i.e. related by a similarity transformation $S$ as $B=S A S^{-1}$. Then, using Eqs. (A3), (A8), and (A9), we obtain

$$
\operatorname{adj} B=S(\operatorname{adj} A) S^{-1} .
$$

Let the quadratic matrix $A$ be regular and $B$ be the matrix with elements $B_{i j}=\lambda_{i} A_{i j} \quad\left(B_{i j}=A_{i j} \lambda_{j}\right)$. If $\lambda_{i} \neq 0$ for $i=1, \ldots, n$, then $B$ is regular, and its inverse $B^{-1}$ has elements

$$
\left(B^{-1}\right)_{i j}=\left(A^{-1}\right)_{i j} \frac{1}{\lambda_{j}}\left(\left(B^{-1}\right)_{i j}=\frac{1}{\lambda_{i}}\left(A^{-1}\right)_{i j}\right) .
$$

This may be understood by observing that the inverse $\Lambda^{-1}$ of the matrix $\Lambda$ with elements $\Lambda_{i j}=\lambda_{i} \delta_{i j}$ has elements $\left(\Lambda^{-1}\right)_{i j}=\left(1 / \lambda_{i}\right) \delta_{i j}$.

Let the quadratic matrix $A$ be a function of some variable $x$. If $A$ is differentiable with respect to $x$, then we may use Jacobi's formula,

$$
(\operatorname{det} A)^{\prime}=\operatorname{tr}\left[(\operatorname{adj} A) A^{\prime}\right],
$$

where the prime indicates differentiation with respect to $x$. This follows from Eq. (A1) and the identity 


$$
\operatorname{det} A=\exp (\operatorname{tr} \ln A) \text {. }
$$

To show this, we first observe that

$$
\operatorname{tr} \ln A=\operatorname{tr} \ln \left[I_{n}-\left(I_{n}-A\right)\right]=-\sum_{k=1}^{\infty} \frac{1}{k} \operatorname{tr}\left(I_{n}-A\right)^{k} .
$$

Differentiating Eq. (A14), we obtain

$$
\begin{aligned}
(\operatorname{tr} \ln A)^{\prime} & =\sum_{k=1}^{\infty} \operatorname{tr}\left[\left(I_{n}-A\right)^{k-1} A^{\prime}\right]=\operatorname{tr}\left[\sum_{k=0}^{\infty}\left(I_{n}-A\right)^{k} A^{\prime}\right] \\
& =\operatorname{tr}\left[\frac{1}{I_{n}-\left(I_{n}-A\right)} A^{\prime}\right] \\
& =\operatorname{tr}\left(A^{-1} A^{\prime}\right) .
\end{aligned}
$$

Differentiating Eq. (A13), we thus obtain

$$
\begin{aligned}
(\operatorname{det} A)^{\prime} & =(\operatorname{det} A)(\operatorname{tr} \ln A)^{\prime}=(\operatorname{det} A) \operatorname{tr}\left(A^{-1} A^{\prime}\right) \\
& =\operatorname{tr}\left[(\operatorname{adj} A) A^{\prime}\right],
\end{aligned}
$$

where we have used Eq. (A15) in the second equality and Eq. (A8) in the third one. We note that Eq. (A12) also holds true if $\operatorname{det} A=0$ at the point $x$ at which the derivative is taken. If $A$ is regular, then

$$
\left(A^{-1}\right)^{\prime}=-A^{-1} A^{\prime} A^{-1} .
$$

This follows by differentiating $A A^{-1}=A^{-1} A=I_{n}$ and solving for $\left(A^{-1}\right)^{\prime}$.

\section{APPENDIX B: DIRAC SPINORS OF UNSTABLE FERMIONS}

In this appendix, we introduce Dirac spinors for unstable fermions and discuss their properties. We work in the Dirac representation of the $\gamma$ matrices, listed e.g. in Eq. (A-22) of Ref. [34], in which

$$
\gamma^{0}=\left(\begin{array}{cc}
I_{2} & 0 \\
0 & -I_{2}
\end{array}\right), \quad \gamma^{i}=\left(\begin{array}{cc}
0 & \sigma^{i} \\
-\sigma^{i} & 0
\end{array}\right),
$$

where $\sigma^{i}$ with $i=1,2,3$ are the Pauli matrices. The $\gamma$ matrices and the Dirac spinors constructed by using them may be converted to any other equivalent representation via an appropriate unitary transformation.

We consider an unstable Dirac fermion with pole mass $M$ and its antiparticle. We denote their four-component Dirac spinors by $u(\vec{p}, M, s)$ and $v(\vec{p}, M, s)$, respectively, where $\vec{p}$ is the three-momentum and $s= \pm 1 / 2$ is the eigenvalue of the designated component of the spin operator. On-shellness, $p^{2}=M^{2}$, is implied, and hence the energy is

$$
p^{0}=\sqrt{\vec{p}^{2}+M^{2}} .
$$

The Dirac spinors $u(\vec{p}, M, s)$ and $v(\vec{p}, M, s)$ must satisfy the Dirac equations

$$
(\not p-M) u(\vec{p}, M, s)=(\not p+M) v(\vec{p}, M, s)=0 .
$$

The solutions may be represented as

$$
\begin{aligned}
& u(\vec{p}, M, s)=\frac{\not p+M}{\sqrt{p^{0}+M}}\left(\begin{array}{c}
\phi(s) \\
0
\end{array}\right), \\
& v(\vec{p}, M, s)=\frac{-\not p+M}{\sqrt{p^{0}+M}}\left(\begin{array}{c}
0 \\
\chi(s)
\end{array}\right),
\end{aligned}
$$

where $\phi(s)$ and $\chi(s)$ are two-component Pauli spinors, which have no functional dependencies on $\vec{p}$ and $M$. The latter are normalized as

$$
\phi^{\dagger}(s) \phi\left(s^{\prime}\right)=\chi^{\dagger}(s) \chi\left(s^{\prime}\right)=\delta_{s s^{\prime}},
$$

and satisfy the completeness relations

$$
\sum_{s=-1 / 2}^{1 / 2} \phi(s) \otimes \phi^{\dagger}(s)=\sum_{s=-1 / 2}^{1 / 2} \chi(s) \otimes \chi^{\dagger}(s)=I_{2} .
$$

If the spin quantization axis is chosen along the flight direction, then the Pauli spinors $\phi(s)$ and $\chi(s)$ satisfy the eigenvalue equations,

$$
\frac{\vec{p}}{|\vec{p}|} \cdot \frac{\vec{\sigma}}{2} \phi(s)=s \phi(s), \quad \frac{\vec{p}}{|\vec{p}|} \cdot \frac{\vec{\sigma}}{2} \chi(s)=-s \chi(s),
$$

so as to represent states of definite helicity.

Naively taking the usual Hermitian conjugate of Eq. (B3), we obtain

$$
\bar{u}\left(\vec{p}^{*}, M^{*}, s\right)\left(\not p^{*}-M^{*}\right)=\bar{v}\left(\vec{p}^{*}, M^{*}, s\right)\left(\not p^{*}+M^{*}\right)=0,
$$

where $p^{*}=p_{\mu}^{*} \gamma^{\mu}$ and

$$
\begin{aligned}
\bar{u}\left(\vec{p}^{*}, M^{*}, s\right) & =[u(\vec{p}, M, s)]^{\dagger} \gamma^{0}, \\
\bar{v}\left(\vec{p}^{*}, M^{*}, s\right) & =[v(\vec{p}, M, s)]^{\dagger} \gamma^{0} .
\end{aligned}
$$

The antispinors thus defined are unsuitable for the construction of fermionic quantum fields because they do not complement Eq. (B4) so as to yield the correct normalization and completeness relations. In this sense, they are not actually the adjoint spinors with respect to $u(\vec{p}, M, s)$ and $v(\vec{p}, M, s)$. 
Instead, we take the antispinors to be $\bar{u}(\vec{p}, M, s)$ and $\bar{v}(\vec{p}, M, s)$, which emerge from $\bar{u}\left(\vec{p}^{*}, M^{*}, s\right)$ and $\bar{v}\left(\vec{p}^{*}, M^{*}, s\right)$ defined in Eq. (B9) by replacing everywhere $\vec{p}^{*}$ and $M^{*}$ with $\vec{p}$ and $M$ and hence also $p^{0 *}$ with $p^{0}$. We then recover all the relations familiar from the Dirac spinors of stable fermions, including the Dirac equations,

$$
\bar{u}(\vec{p}, M, s)(\not p-M)=\bar{v}(\vec{p}, M, s)(\not p+M)=0,
$$

the normalizations,

$\bar{u}(\vec{p}, M, s) u\left(\vec{p}, M, s^{\prime}\right)=-\bar{v}(\vec{p}, M, s) v\left(\vec{p}, M, s^{\prime}\right)=2 M \delta_{s s^{\prime}}$, $\bar{v}(\vec{p}, M, s) u\left(\vec{p}, M, s^{\prime}\right)=\bar{u}(\vec{p}, M, s) v\left(\vec{p}, M, s^{\prime}\right)=0$,

and the completeness relations,

$$
\begin{aligned}
& \sum_{s=-1 / 2}^{1 / 2} u(\vec{p}, M, s) \otimes \bar{u}(\vec{p}, M, s)=p+M, \\
& \sum_{s=-1 / 2}^{1 / 2} v(\vec{p}, M, s) \otimes \bar{v}(\vec{p}, M, s)=\not p-M .
\end{aligned}
$$

Armed with such appropriate definitions of Dirac spinors and antispinors, we may then proceed to construct the fermionic quantum fields in the noninteracting theory,

$$
\begin{aligned}
\psi(x)= & \int \frac{d^{3} p}{(2 \pi)^{3} 2 p^{0}} \sum_{s=-1 / 2}^{1 / 2}\left[a(\vec{p}, s) u(\vec{p}, M, s) e^{-i p \cdot x}\right. \\
& \left.+b^{\dagger}(\vec{p}, s) v(\vec{p}, M, s) e^{i p \cdot x}\right], \\
\bar{\psi}(x)= & \int \frac{d^{3} p}{(2 \pi)^{3} 2 p^{0}} \sum_{s=-1 / 2}^{1 / 2}\left[b(\vec{p}, s) \bar{v}(\vec{p}, M, s) e^{-i p \cdot x}\right. \\
& \left.+a^{\dagger}(\vec{p}, s) \bar{u}(\vec{p}, M, s) e^{i p \cdot x}\right],
\end{aligned}
$$

where $\vec{p}$ is taken to be real, Eq. (B2) is implied, and $a(\vec{p}, s), a^{\dagger}(\vec{p}, s), b(\vec{p}, s)$, and $b^{\dagger}(\vec{p}, s)$ are the annihilation and creation operators of the Dirac fermion and antifermion. The latter satisfy the anticommutation relations

$$
\begin{aligned}
& \left\{a(\vec{p}, s), a^{\dagger}\left(\vec{p}^{\prime}, s^{\prime}\right)\right\} \\
& \quad=\left\{b(\vec{p}, s), b^{\dagger}\left(\vec{p}^{\prime}, s^{\prime}\right)\right\}=(2 \pi)^{3} 2 p^{0} \delta^{(3)}\left(\vec{p}-\vec{p}^{\prime}\right) \delta_{s s^{\prime}}, \\
& \left\{a(\vec{p}, s), b\left(\vec{p}^{\prime}, s^{\prime}\right)\right\} \\
& \quad=\left\{a(\vec{p}, s), b^{\dagger}\left(\vec{p}^{\prime}, s^{\prime}\right)\right\}=\left\{a^{\dagger}(\vec{p}, s), b\left(\vec{p}^{\prime}, s^{\prime}\right)\right\} \\
& \quad=\left\{a^{\dagger}(\vec{p}, s), b^{\dagger}\left(\vec{p}^{\prime}, s^{\prime}\right)\right\}=0 .
\end{aligned}
$$

We note that $\bar{\psi}(x)$ emerges from $[\psi(x)]^{\dagger} \gamma^{0}$ by undoing the complex conjugation of $M$. This is in line with the observations made in the context of Eqs. (B8) and (B9) and is required in order not to spoil essential relationships and to reproduce the Feynman propagator in its correct form. In fact, we thus recover the canonical equal-time anticommutation relations,

$$
\begin{aligned}
& \left\{\psi(t, \vec{x}), \bar{\psi}\left(t, \vec{x}^{\prime}\right)\right\}=\gamma^{0} \delta^{(3)}\left(\vec{x}-\vec{x}^{\prime}\right), \\
& \left\{\psi(t, \vec{x}), \psi\left(t, \vec{x}^{\prime}\right)\right\}=\left\{\bar{\psi}(t, \vec{x}), \bar{\psi}\left(t, \vec{x}^{\prime}\right)\right\}=0,
\end{aligned}
$$

the Wick contractions,

$$
\begin{aligned}
\langle 0|T[\psi(x) \bar{\psi}(y)]| 0\rangle & =\int \frac{d^{4} p}{(2 \pi)^{4}} e^{-i p \cdot(x-y)} \frac{i}{p-M}, \\
\langle 0|T[\psi(x) \psi(y)]| 0\rangle & =\langle 0|T[\bar{\psi}(x) \bar{\psi}(y)]| 0\rangle=0,
\end{aligned}
$$

and the Feynman propagator in momentum space,

$i P(\not p)=\int d^{4} x e^{i p \cdot x}\langle 0|T[\psi(x) \bar{\psi}(0)]| 0\rangle=\frac{i}{\not p-M}$,

in the forms familiar from the case of stable Dirac fermions.

If $\vec{p}$ is real on mass shell, then we glean from Eqs. (14) and (B2) that

$$
\begin{aligned}
\operatorname{Re} p^{0} & =\sqrt{1 / 2\left(\sqrt{x^{2}+y^{2}}+x\right)}=\sqrt{x}\left[1+\frac{y^{2}}{8 x^{2}}+\mathcal{O}\left(\frac{y^{4}}{x^{4}}\right)\right], \\
\operatorname{Im} p^{0} & =-\sqrt{1 / 2\left(\sqrt{x^{2}+y^{2}}-x\right)} \\
& =-\frac{y}{2 \sqrt{x}}\left[1-\frac{y^{2}}{8 x^{2}}+\mathcal{O}\left(\frac{y^{4}}{x^{4}}\right)\right],
\end{aligned}
$$

where $x=\vec{p}^{2}+m^{2}-\Gamma^{2} / 4$ and $y=m \Gamma$. The fact that $\operatorname{Im} p^{0}$ is finite and negative implies that Eq. (B13) is only well defined in a bounded interval of time $x^{0}$. However, there are no such restrictions for Eqs. (B15) and (B16). Of course, the reality of $\vec{p}$ is, in general, not preserved under Lorentz transformations, which limits the usefulness of Eq. (B13). The choice of four-momentum $p=M u$ [35], where $u$ is the (real) four-velocity, would restore Lorentz covariance, but render Eq. (B13) ill-defined, except at the origin $x=0$. 
[1] G. Aad et al. (ATLAS Collaboration), Phys. Lett. B 716, 1 (2012); S. Chatrchyan et al. (CMS Collaboration), Phys. Lett. B 716, 30 (2012).

[2] J. Beringer et al. (Particle Data Group), Phys. Rev. D 86, 010001 (2012).

[3] F. Bezrukov, M. Yu. Kalmykov, B. A. Kniehl, and M. Shaposhnikov, J. High Energy Phys. 10 (2012) 140; S. Alekhin, A. Djouadi, and S. Moch, Phys. Lett. B 716, 214 (2012).

[4] G.'t Hooft, Nucl. Phys. B33, 173 (1971); J. C. Taylor, Nucl. Phys. B33, 436 (1971); G.'t Hooft, Nucl. Phys. B35, 167 (1971); B. W. Lee, Phys. Rev. D 5, 823 (1972); G.'t Hooft and M. Veltman, Nucl. Phys. B50, 318 (1972); D. A. Ross and J. C. Taylor, Nucl. Phys. B51, 125 (1973); B58, 643(E) (1973).

[5] J. Fleischer and F. Jegerlehner, Phys. Rev. D 23, 2001 (1981); M. Böhm, H. Spiesberger, and W. Hollik, Fortschr. Phys. 34, 687 (1986).

[6] K.-i. Aoki, Z. Hioki, R. Kawabe, M. Konuma, and T. Muta, Prog. Theor. Phys. Suppl. 73, 1 (1982).

[7] W. F. L. Hollik, Fortschr. Phys. 38, 165 (1990).

[8] A. Sirlin, Phys. Rev. D 22, 971 (1980).

[9] P. A. Grassi, Nucl. Phys. B462, 524 (1996); E. Kraus and K. Sibold, Nucl. Phys. B, Proc. Suppl. 51C, 81 (1996); E. Kraus, Ann. Phys. (N.Y.) 262, 155 (1998); P. A. Grassi, T. Hurth, and M. Steinhauser, Ann. Phys. (N.Y.) 288, 197 (2001).

[10] N. Cabibbo, Phys. Rev. Lett. 10, 531 (1963); M. Kobayashi and T. Maskawa, Prog. Theor. Phys. 49, 652 (1973).

[11] B. Pontecorvo, Zh. Eksp. Teor. Fiz. 33, 549 (1957) [Sov. Phys. JETP 6, 429 (1958)]; Zh. Eksp. Teor. Fiz. 34, 247 (1958) [Sov. Phys. JETP 7, 172 (1958)]; Z. Maki, M. Nakagawa, and S. Sakata, Prog. Theor. Phys. 28, 870 (1962); B. Pontecorvo, Zh. Eksp. Teor. Fiz. 53, 1717 (1967) [Sov. Phys. JETP 26, 984 (1968)].

[12] A. Denner and T. Sack, Nucl. Phys. B347, 203 (1990); P. Gambino, P. A. Grassi, and F. Madricardo, Phys. Lett. B 454, 98 (1999); A. Barroso, L. Brücher, and R. Santos, Phys. Rev. D 62, 096003 (2000); D. Espriu and J. Manzano, Phys. Rev. D 63, 073008 (2001); D. Espriu, in Proceedings of the 12th International Seminar on High-Energy Physics (QUARKS 2002), Novgorod, Russia, 1-7 June 2002, edited by V. A. Matveev, V. A. Rubakov, S. M. Sibiryakov, and A. N. Tavkhelidze (INR, Moscow, 2002); Y. Yamada, Phys. Rev. D 64, 036008 (2001); A. Pilaftsis, Phys. Rev. D 65, 115013 (2002); Y. Zhou, Phys. Lett. B 577, 67 (2003); J. Phys. G 30, 491 (2004); Y. Liao, Phys. Rev. D 69, 016001 (2004); A. Denner, E. Kraus, and M. Roth, Phys. Rev. D 70, 033002 (2004).
[13] K.-P. O. Diener and B. A. Kniehl, Nucl. Phys. B617, 291 (2001).

[14] B. A. Kniehl and A. Sirlin, Phys. Rev. Lett. 97, 221801 (2006); Phys. Rev. D 74, 116003 (2006); Phys. Lett. B 673, 208 (2009); AIP Conf. Proc. 1182, 327 (2009).

[15] B. A. Kniehl and A. Pilaftsis, Nucl. Phys. B474, 286 (1996).

[16] A. A. Almasy, B. A. Kniehl, and A. Sirlin, Nucl. Phys. B818, 115 (2009).

[17] B. A. Kniehl, F. Madricardo, and M. Steinhauser, Phys. Rev. D 62, 073010 (2000); A. A. Almasy, B. A. Kniehl, and A. Sirlin, Phys. Rev. D 79, 076007 (2009); 82, 059901(E) (2010).

[18] A. A. Almasy, B. A. Kniehl, and A. Sirlin, Phys. Rev. D 83, 096004 (2011).

[19] P. Gambino and P. A. Grassi, Phys. Rev. D 62, 076002 (2000).

[20] N. K. Nielsen, Nucl. Phys. B101, 173 (1975).

[21] B. A. Kniehl and A. Sirlin, Phys. Rev. D 85, 036007 (2012).

[22] H. Lehmann, K. Symanzik, and W. Zimmermann, Nuovo Cimento 1, 205 (1955); 6, 319 (1957).

[23] B. A. Kniehl, Phys. Rev. Lett. 112, 071603 (2014).

[24] F. J. Dyson, Phys. Rev. 75, 1736 (1949).

[25] D. Espriu, J. Manzano, and P. Talavera, Phys. Rev. D 66, 076002 (2002).

[26] J. F. Donoghue, Phys. Rev. D 19, 2772 (1979).

[27] M. C. Smith and S. S. Willenbrock, Phys. Rev. Lett. 79, 3825 (1997); M. Passera and A. Sirlin, Phys. Rev. D 58, 113010 (1998).

[28] B. A. Kniehl and A. Sirlin, Phys. Rev. D 77, 116012 (2008).

[29] A. Sirlin and R. Zucchini, Nucl. Phys. B266, 389 (1986); G. Degrassi and A. Sirlin, Nucl. Phys. B383, 73 (1992).

[30] R. Hempfling and B. A. Kniehl, Phys. Rev. D 51, 1386 (1995); F. Jegerlehner and M. Yu. Kalmykov, Nucl. Phys. B676, 365 (2004); M. Faisst, J. H. Kühn, and O. Veretin, Phys. Lett. B 589, 35 (2004); B. A. Kniehl, J. H. Piclum, and M. Steinhauser, Nucl. Phys. B695, 199 (2004); F. Jegerlehner, M. Yu. Kalmykov, and B. A. Kniehl, Phys. Lett. B 722, 123 (2013); Proc. Sci., DIS (2013) 190 [arXiv:1307.4226]; B. A. Kniehl and O.L. Veretin, arXiv:1401.1844 [Nucl. Phys. B (in preparation)].

[31] W. A. Bardeen, A. J. Buras, D. W. Duke, and T. Muta, Phys. Rev. D 18, 3998 (1978).

[32] C. G. Bollini and J. J. Giambiagi, Nuovo Cimento Soc. Ital. Fis. 12B, 20 (1972); G.'t Hooft and M. Veltman, Nucl. Phys. B44, 189 (1972); J. F. Ashmore, Lett. Nuovo Cimento 4, 289 (1972); Commun. Math. Phys. 29, 177 (1973).

[33] B. A. Kniehl, arXiv:1404.5908.

[34] C. Itzykson and J.-B. Zuber, Quantum Field Theory (McGraw-Hill, New York, 1980).

[35] A. Sirlin (private communication). 